\title{
21. VOLCANIC ASH LAYERS FROM SITES 828, 830, 831, 832, AND 833, NEW HEBRIDES ISLAND ARC ${ }^{1}$
}

\author{
Peter E. Baker, ${ }^{2}$ Massimo Coltorti, ${ }^{3}$ Louis Briqueu, ${ }^{4}$ Toshiaki Hasenaka, ${ }^{5}$ and Eric Condliffe ${ }^{2}$
}

\begin{abstract}
Volcanic ash layers (1-3 cm thick) are abundant in the North Aoba Basin drill sites but less common at forearc sites. Ash deposited on the forearc slopes is liable to be redistributed as turbidites. In addition, the westerly upper winds also minimize ash-fall on the western (forearc) side of the New Hebrides Island Arc.

Crystalline components in the ashes are primarily plagioclase $\left(\mathrm{An}_{90}-\mathrm{An}_{44}\right)$, clinopyroxene $\left(\mathrm{Ca}_{46} \mathrm{Mg}_{49} \mathrm{Fe}_{5}-\mathrm{Ca}_{43} \mathrm{Mg}_{33} \mathrm{Fe}_{24}\right)$, olivine $\left(\mathrm{Fo}_{87}-\mathrm{Fo}_{62}\right)$, and titanomagnetite. There are also small amounts of orthopyroxene, magnetite, apatite, and quartz. Glass shards occur in most of the ashes and range in composition from basalt to rhyolite. There is often a variety of glass compositions within a single ash layer. One explanation for this is that the rate of accumulation of ash from several different eruptions or eruptive phases exceeded the background sedimentation rate: there may also have been a certain amount of reworking. The high- $\mathrm{K}$ and low-K trends previously recognized in volcanic rocks from the New Hebrides Island Arc are clearly represented in the Leg 134 glasses. All of the ashes investigated here are thought to have originated from the Central Chain volcanoes. The source of the high-K group was probably the Central Basin volcanoes of Santa Maria, Aoba, and Ambrym. The lower-K series includes a distinctive group of dacites and is likely to have originated from the Epi-Tongoa-Tongariki sector of the arc where major pyroclastic eruptions, associated with caldera collapse, have occurred during the Holocene, perhaps as recently as $400 \mathrm{yr}$ ago.
\end{abstract}

\section{INTRODUCTION}

Volcanic ash layers, usually $<2 \mathrm{~cm}$ thick, were encountered at most of the Leg 134 sites (Fig. 1). Although the ash layers are abundant in the North Aoba Basin, they are less common at the forearc sites because Sites 832 and 833 in the North Aoba Basin are closer to the active volcanoes of the Central Chain, and the distribution of pyroclast fall deposits was largely controlled by the prevailing winds. Although the southeast trades are the main influence at lower altitudes $(<2 \mathrm{~km})$, strong westerlies dominate above this, to a height of about $18 \mathrm{~km}$ (Eade and Gregory, 1992). The ash distribution was influenced by these upper westerly winds and the effect minimized accumulation at the forearc sites. The latter was affected only by eruptions of exceptional magnitude or during atypical wind conditions.

There are also differences in the incidence of ash layers between different forearc sites. Some ash is preserved at Site 828 on the North d'Entrecasteaux Ridge (NDR) and at Site 831 on Bougainville Guyot, part of the South d'Entrecasteaux Chain (SDC; Fig. 1). These provide relatively upstanding and stable sites on the subducting AustraliaIndia Plate. In contrast, primary ash-fall layers are apparently absent from Sites 827 and 829 , both of which are situated on the steep forearc slope between Espiritu Santo Island and the New Hebrides Trench (Fig. 1). The upper parts of both sections are composed almost entirely of greenish clayey-volcanic silt, siltstone, and sandstone, including many turbidites and slump features. Any ash deposited in this highly unstable environment has probably been reworked downslope. Site 830 lies in a similar position with respect to the trench, but the arc slope to the east is less steep: volcanic silts again prevail in lithostratigraphic Unit I (0-174.9 mbsf), but there are occasional ash layers in the uppermost part.

\footnotetext{
${ }^{1}$ Greene, H.G., Collot, J.-Y., Stokking, L.B., et al., 1994. Proc. ODP, Sci. Results, 134: College Station, TX (Ocean Drilling Program).

${ }^{2}$ Department of Earth Sciences, University of Leeds, Leeds LS2 9JT, United Kingdom.

${ }_{3}^{3}$ Mineralogy Institute, Ferrara University, C.so E. 1 d'Este 32, 44100, Ferrara, Italy.

${ }^{4}$ C.N.R.S. France, Laboratoire de Géochimie Isotopique, U.S.T.L., C.P. 066, 34095 Montpellier Cedex 2, France.

${ }^{5}$ Institute of Mineralogy, Petrology and Economic Geology, Faculty of Science, Tohoku University, Aoba, Sendai, Miyagi 980, Japan.
}

\section{ANALYTICAL METHODS}

The major element chemistry of minerals and glass shards was determined with a CAMECA SX-50 electron microprobe located in the Electron Microscopy Laboratory at Leeds University. The instrument is fitted with three wavelength dispersive spectrometers and a LINK 10/55S energy dispersive system. Analysis conditions were as follows:

1. Silicates and opaque oxides, excluding feldspars-beam energy $15 \mathrm{kV}$; beam current $15 \mathrm{na}$; count times: $\mathrm{Na}, \mathrm{Mg}, \mathrm{Al}, \mathrm{Si}, \mathrm{K}, \mathrm{Ca}$, Ti: all $15 \mathrm{~s}$ on peak, $10 \mathrm{~s}$ on background; $\mathrm{Cr}, \mathrm{Mn}, \mathrm{Fe}, \mathrm{Ni}: 30 \mathrm{~s}$ on peak, $20 \mathrm{~s}$ on background.

2. Feldspars and glasses-beam energy $15 \mathrm{kV}$; beam current 10 $\mathrm{na}$; count times for all elements: $10 \mathrm{~s}$ on peak, $10 \mathrm{~s}$ on background. Where necessary, beam broadened to 2 or $3 \mu \mathrm{m}$ to prevent excessive element loss.

3. Raw counts were corrected for interelemental effects using CAMECA proprietary software.

4. Standards used: Na-albite; $\mathrm{Mg}$-spinel; Al-kyanite; Si-diopside; $\mathrm{K}$-orthoclase; $\mathrm{Ca}$-wollastonite; $\mathrm{Ti}$-sphene; $\mathrm{Cr}$-chromite; $\mathrm{Mn}$ rhodonite; $\mathrm{Fe}$-hematite; $\mathrm{Ni}$-nickel oxide.

Problems associated with alkali metal loss from hydrated glasses during electron beam bombardment have been discussed by Arculus and Bloomfield (1992). In the present study, Na was calibrated on albite using a $2 \mu \mathrm{m}$ diameter beam; $\mathrm{Na}$ and $\mathrm{K}$ were analyzed first in the sequence of determinations on volcanic glasses. Tests on homogeneous shards of basaltic glass have shown that the $\mathrm{Na}$ and $\mathrm{K}$ count rates are stable for up to $50 \mathrm{~s}$ at $10 \mathrm{nA}$ beam current, provided the beam diameter is $2 \mu \mathrm{m}$ or more: the narrow width of most shards generally precluded widening the beam beyond this. In addition, a comparison of $\mathrm{Na}$ and $\mathrm{K}$ analyzed using EDS conditions (50-100 s live time, $2 \mathrm{nA}$ beam current, $2 \mu \mathrm{m}, 5 \mu \mathrm{m}$ and $10 \mu \mathrm{m}$ diameter beam) with these elements analyzed at $10 \mathrm{nA}$ for $10 \mathrm{~s}$ with similar beam diameters, indicates that there is no significant $\mathrm{Na}$ or $\mathrm{K}$ loss at the higher beam currents and short count times employed in this study. Using the same conditions reported here, Leeds analyses of glass JDF-D2 from Lamont-Doherty Earth Observatory (formerly Lamont-Doherty Geological Observatory) show good agreement with accepted $\mathrm{Na}_{2} \mathrm{O}$ values. 


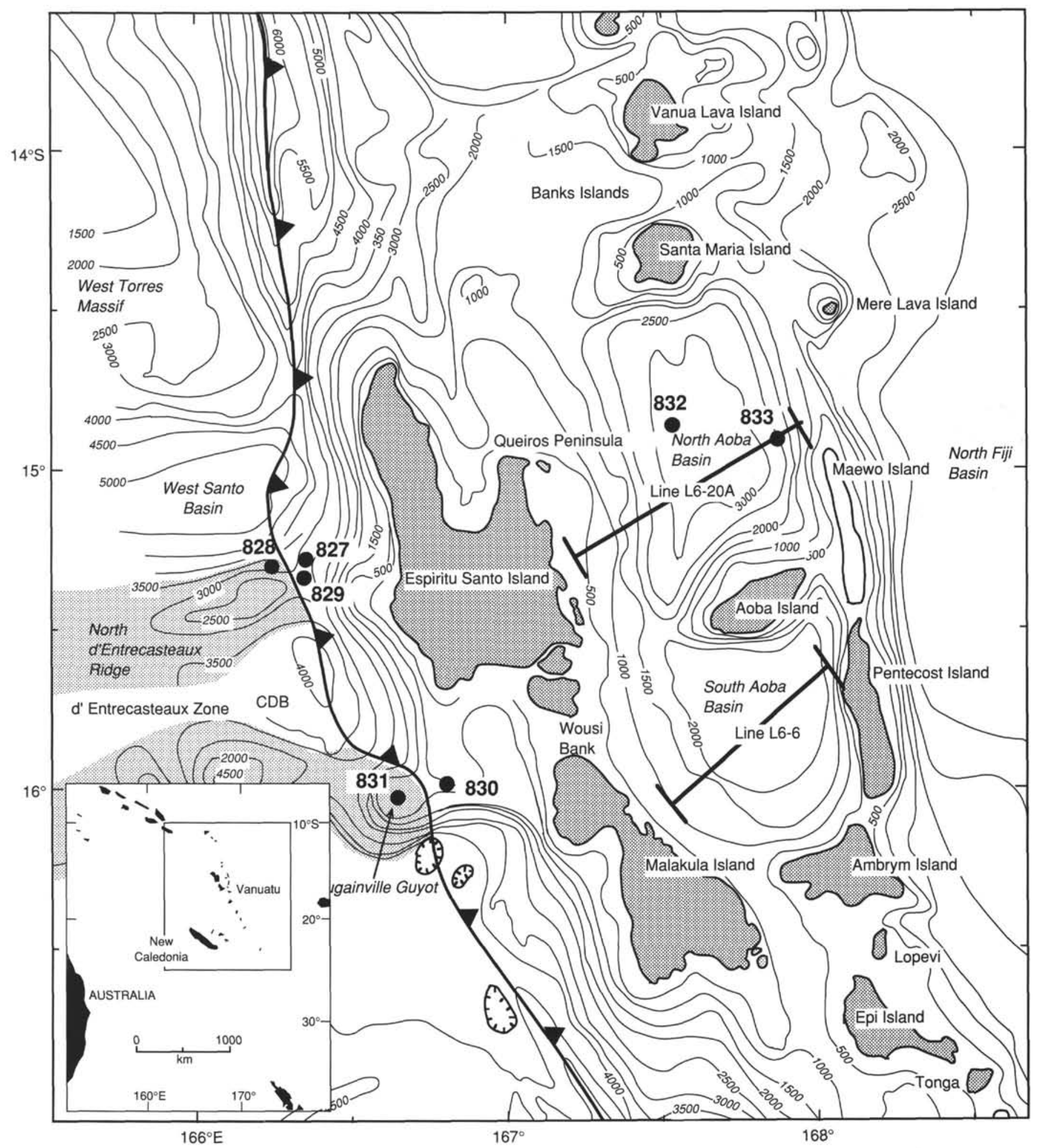

Figure 1. Location map of Leg 134 sites and nearby islands. 


\section{SAMPLE DESCRIPTIONS}

\section{4-828A-2H-6, 7-9 cm}

A volcanic ash containing two types of glass, large, clear fragments of more basaltic composition ( $50 \mathrm{wt} \% \mathrm{SiO}_{2}$ ), and a more differentiated glass $\left(55 \mathrm{wt} \% \mathrm{SiO}_{2}\right)$ packed with feldspar crystallites. It also contains discrete crystals of olivine $\left(\mathrm{Fo}_{87}\right)$, plagioclase $\left(\mathrm{An}_{79-63}\right)$, and clinopyroxene zoned in the range $\mathrm{Ca}_{41} \mathrm{Mg}_{46} \mathrm{Fe}_{13}$ to $\mathrm{Ca}_{45} \mathrm{Mg}_{35} \mathrm{Fe}_{20}$. Calcite grains and shell fragments are also present in this band.

\section{4-828A-4H-2, 55-57 cm}

A volcanic ash with more uniform basaltic glass $\left(52 \mathrm{wt} \% \mathrm{SiO}_{2}\right)$, containing phenocrysts of clinopyroxene $\left(\mathrm{Ca}_{45} \mathrm{Mg}_{41} \mathrm{Fe}_{14}\right)$, and plagioclase $\left(\mathrm{An}_{83-66}\right)$.

\section{4-830A-1H-3, 75-76 cm}

A fragment of pumice recovered from calcareous volcanic silt. It is a highly vesicular, cellular dacitic glass $\left(67 \mathrm{wt} \% \mathrm{SiO}_{2}, 3.8 \mathrm{wt} \%\right.$ $\mathrm{K}_{2} \mathrm{O}$ ) with sparse crystals of plagioclase $\left(\mathrm{An}_{46}\right)$ and orthopyroxene $\left(\mathrm{Ca}_{4} \mathrm{Mg}_{59} \mathrm{Fe}_{37}\right)$.

\section{4-830A-3H-3, 75-76 cm}

A rhyodacite ash with rare plagioclase crystals $\left(\mathrm{An}_{82}\right)$. Recalculated volatile-free, the sample has $69.9 \mathrm{wt} \% \mathrm{SiO}_{2}$ and $4.39 \mathrm{wt} \% \mathrm{~K}_{2} \mathrm{O}$, placing it close to the boundary of the high- $\mathrm{K}$ calc-alkaline and shoshonitic series, rather similar to the ashes from Santa Maria Island.

\section{$134-830 \mathrm{~A}-3 \mathrm{H}-4,108-110 \mathrm{~cm}$}

A reworked ash, lacking fresh glass and containing a considerable amount of calcite and clay minerals. The crystalline components are clinopyroxene ranging from $\mathrm{Ca}_{47} \mathrm{Mg}_{48} \mathrm{Fe}_{5}$ to $\mathrm{Ca}_{42} \mathrm{Mg}_{41} \mathrm{Fe}_{17}$. Plagioclase is zoned from $\mathrm{An}_{90}$ to $\mathrm{An}_{55}$ and the opaques are titanomagnetite.

\section{4-831A-3H6, 54-55 cm}

Ash containing two glass compositions, one with $52.6 \mathrm{wt} \% \mathrm{SiO}_{2}$ and $1.3 \mathrm{wt} \% \mathrm{~K}_{2} \mathrm{O}$ and the other with $55.2 \mathrm{wt} \% \mathrm{SiO}_{2}$ and $1.6 \mathrm{wt} \% \mathrm{~K}_{2} \mathrm{O}$. Larger isolated plagioclase crystals have a composition of $\mathrm{An}_{76}$ and plagioclase laths in the glass range from $\mathrm{An}_{58}$ to $\mathrm{An}_{44}$. Opaque grains are magnetite.

\section{4-832A-1H-4, 54-56 cm}

A composite volcanic ash with a fairly wide span of glass compositions which nevertheless tend to concentrate in two groups. The more basic one has about $51 \mathrm{wt} \% \mathrm{SiO}_{2}$ (volatile-free basis) and about $2 \mathrm{wt} \%$ $\mathrm{K}_{2} \mathrm{O}$, and the other is andesitic with $58-60 \mathrm{wt} \% \mathrm{SiO}_{2}$ and around $4 \mathrm{wt} \%$ $\mathrm{K}_{2} \mathrm{O}$. Plagioclase crystals have a compositional range of $\mathrm{An}_{79}$ to $\mathrm{An}_{58}$. The ash also contains olivine $\left(\mathrm{Fo}_{77-65}\right)$, clinopyroxene $\left(\mathrm{Ca}_{46} \mathrm{Mg}_{49} \mathrm{Fe}_{5}\right.$ to $\left.\mathrm{Ca}_{43} \mathrm{Mg}_{33} \mathrm{Fe}_{24}\right)$, and sparse orthopyroxene $\left(\mathrm{Ca}_{5} \mathrm{Mg}_{70} \mathrm{Fe}_{25}\right)$. Other constituents are titanomagnetite and apatite.

\section{4-832A-3H-1, 36-38 cm}

Ash layer with a wide range of glass compositions from $54 \mathrm{wt} \%$ $\mathrm{SiO}_{2}$ and 2.8 wt $\% \mathrm{~K}_{2} \mathrm{O}$ to 61 wt $\% \mathrm{SiO}_{2}$ and $4.6 \mathrm{wt} \% \mathrm{~K}_{2} \mathrm{O}$ on a volatile-free basis. Shards and clasts of glass are invariably fresh and there is no doubt that they represent a real compositional spectrum and are not a function of alteration. Olivine crystals range from $\mathrm{Fo}_{71}$ to $\mathrm{Fo}_{64}$, and clinopyroxene from $\mathrm{Ca}_{46} \mathrm{Mg}_{40} \mathrm{Fe}_{14}$ to $\mathrm{Ca}_{44} \mathrm{Mg}_{37} \mathrm{Fe}_{19}$. Plagioclase is zoned in the range $\mathrm{An}_{79}$ to $\mathrm{An}_{50}$.

\section{4-832A-10H-1, 120-122 cm}

A volcanic ash again showing a range of compositions with the dominant one basaltic: on a volatile-free basis it has $50 \mathrm{wt} \% \mathrm{SiO}_{2}$ and 2.6 wt $\% \mathrm{~K}_{2} \mathrm{O}$. Another glass contains $55 \mathrm{wt} \% \mathrm{SiO}_{2}$ and $1.3 \mathrm{wt} \% \mathrm{~K}_{2} \mathrm{O}$. Athird is a rhyolitic glass with $71 \mathrm{wt} \% \mathrm{SiO}_{2}$ and $4.4 \mathrm{wt} \% \mathrm{~K}_{2} \mathrm{O}$. Plagioclase varies from $\mathrm{An}_{88}$ to $\mathrm{An}_{55}$ and clinopyroxene from $\mathrm{Ca}_{48} \mathrm{Mg}_{44} \mathrm{Fe}_{8}$ to $\mathrm{Ca}_{46} \mathrm{Mg}_{40} \mathrm{Fe}_{14}$. There are also crystals of olivine $\left(\mathrm{Fo}_{62}\right)$.

\section{4-832A-20H-1, 80-82 cm}

A tuff band containing at least two glass compositions. A vesicular scoriaceous glass encloses clinopyroxene crystallites and has $50 \mathrm{wt} \%$ $\mathrm{SiO}_{2}$ and $2.0 \mathrm{wt} \% \mathrm{~K}_{2} \mathrm{O}$. A second glass has $53 \mathrm{wt} \% \mathrm{SiO}_{2}$ and $2.7 \mathrm{wt} \%$ $\mathrm{K}_{2} \mathrm{O}$. Olivine is zoned in the range $\mathrm{Fo}_{86-80}$ and clinopyroxene from $\mathrm{Ca}_{48} \mathrm{Mg}_{45} \mathrm{Fe}_{7}$ to $\mathrm{Ca}_{48} \mathrm{Mg}_{40} \mathrm{Fe}_{12}$. Plagioclase is in the range $\mathrm{An}_{82}$ to $\mathrm{An}_{57}$.

\section{4-833A-1H-6, 72-74 cm}

A composite volcanic ash showing glass compositions ranging between $\mathrm{SiO}_{2} 51.8$ wt $\%, \mathrm{~K}_{2} \mathrm{O} 2.2 \mathrm{wt} \%$; and $\mathrm{SiO}_{2} 56.6$ wt $\%, \mathrm{~K}_{2} \mathrm{O} 4.5$ $\mathrm{wt} \%$, on a volatile-free basis. Clinopyroxene associated with the more basic glass has a composition of $\mathrm{Ca}_{47} \mathrm{Mg}_{41} \mathrm{Fe}_{12}$ and clinopyroxene in a lithic fragment has a composition of $\mathrm{Ca}_{44} \mathrm{Mg}_{38} \mathrm{Fe}_{18}$ : other isolated pyroxenes have compositions intermediate between these two. Olivine $\left(\mathrm{FO}_{73}\right)$ occurs in a lithic fragment and plagioclase ranges from $\mathrm{An}_{76}$ to $\mathrm{An}_{56}$.

\section{MINERAL CHEMISTRY}

Minerals in the Leg 134 ashes occur either as discrete crystals, or enclosed in glass. The latter is the case particularly for some of the plagioclase and clinopyroxene.

Plagioclase is the only feldspar encountered in the ashes and is invariably strongly zoned, usually in oscillatory fashion. Plagioclase compositions tend to be very similar in the majority of the samples studied (Table 1) and the most common range is from $\mathrm{An}_{81}$ to $\mathrm{An}_{57}$. The most calcic composition $\left(\mathrm{An}_{90}\right)$ was found in Sample 134-830A$3 \mathrm{H}-4,108-110 \mathrm{~cm}$.

Clinopyroxene is by far the most abundant of the mafic minerals and shows a very similar compositional spread in ashes from the forearc (Sites 828 and 830) and North Aoba Basin (Sites 832 and 833; Figs. 2 and 3, respectively). Clinopyroxene is almost exclusively augite and ranges between $\mathrm{Ca}_{46} \mathrm{Mg}_{49} \mathrm{Fe}_{5}$ and $\mathrm{Ca}_{43} \mathrm{Mg}_{33} \mathrm{Fe}_{24}$, sometimes extending towards the subcalcic augite field. $\mathrm{Al}_{2} \mathrm{O}_{3}$ content in the clinopyroxene ranges from $2.0 \mathrm{wt} \%$ to $10.7 \mathrm{wt} \%$ and $\mathrm{TiO}_{2}$ from $0.3 \mathrm{wt} \%$ to $1.3 \mathrm{wt} \%$. Some clinopyroxenes from Sample 134-828A$2 \mathrm{H}-6,7-9 \mathrm{~cm}$, (Table 2) are notable for their high contents of $\mathrm{Al}_{2} \mathrm{O}_{3}$ $(<10.7 \mathrm{wt} \%)$ and $\mathrm{TiO}_{2}(<1.3 \mathrm{wt} \%)$. Almost the entire compositional span is represented in single horizons from the forearc (Sample 134-

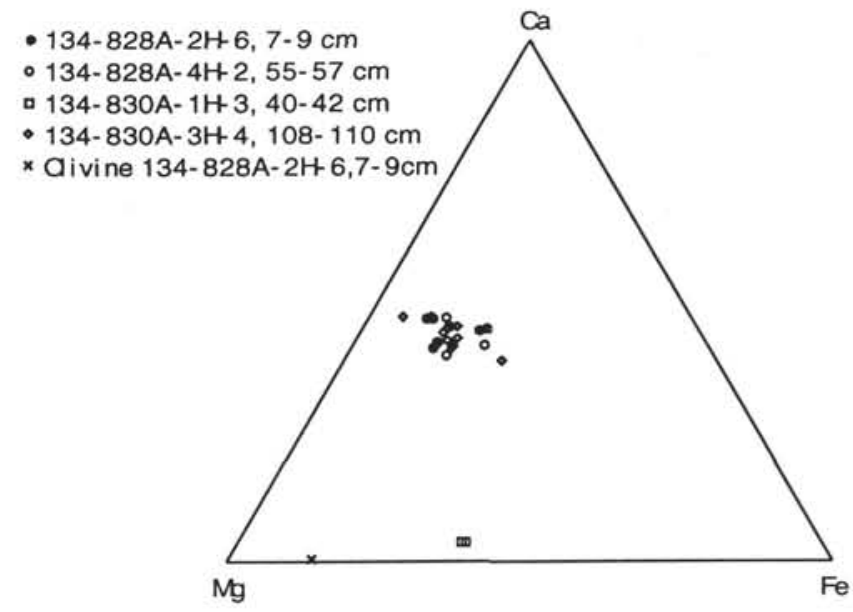

Figure 2. Projections of pyroxene and olivine compositions in terms of relative proportions of $\mathrm{Ca}, \mathrm{Mg}$, and $\mathrm{Fe}$, from forearc Sites 828 and 830 . 
Table 1. Representative microprobe analyses in weight percent $(\mathrm{wt} \%)$ and atomic proportions of plagioclases from Leg 134 ashes. $\mathrm{Total}_{\mathrm{Fe}}$ is reported as $\mathrm{Fe}_{2} \mathrm{O}_{3}$.

\begin{tabular}{|c|c|c|c|c|c|c|c|c|c|c|c|c|c|c|c|c|c|c|c|c|c|c|c|c|}
\hline $\begin{array}{l}\text { Hole: } \\
\text { Core, section: } \\
\text { Interval (cm): }\end{array}$ & $\begin{array}{c}828 \mathrm{~A} \\
2 \mathrm{H}-6 \\
7-9\end{array}$ & $\begin{array}{l}828 \mathrm{~A} \\
4 \mathrm{H}-2 \\
55-57\end{array}$ & $\begin{array}{l}828 \mathrm{~A} \\
4 \mathrm{H}-2 \\
55-57\end{array}$ & $\begin{array}{l}830 \mathrm{~A} \\
1 \mathrm{H}-3 \\
740-42\end{array}$ & $\begin{array}{r}830 \mathrm{~A} \\
1 \mathrm{H}-3 \\
40-42\end{array}$ & $\begin{array}{c}830 \mathrm{~A} \\
3 \mathrm{H}-3 \\
75-76\end{array}$ & $\begin{array}{c}830 \mathrm{~A} \\
3 \mathrm{H}-4 \\
108-110\end{array}$ & $\begin{array}{c}830 \mathrm{~A} \\
3 \mathrm{H}-4 \\
108-110\end{array}$ & $\begin{array}{c}830 \mathrm{~A} \\
3 \mathrm{H}-4 \\
108-110\end{array}$ & $\begin{array}{l}831 \mathrm{~A} \\
3 \mathrm{H}-6 \\
54-55\end{array}$ & $\begin{array}{r}831 \mathrm{~A} \\
3 \mathrm{H}-6 \\
5 \\
54-55\end{array}$ & $\begin{array}{cc} & 832 \mathrm{~A} \\
5 & 1 \mathrm{H}-4 \\
5 & 54-56\end{array}$ & $\begin{array}{r}832 \mathrm{~A} \\
1 \mathrm{H}-4 \\
54-56\end{array}$ & $\begin{array}{c}832 \mathrm{~A} \\
1 \mathrm{H}-4 \\
54-56\end{array}$ & $\begin{array}{l}832 \mathrm{~A} \\
3 \mathrm{H}-1 \\
36-38\end{array}$ & $\begin{array}{ll}832 \mathrm{~A} \\
3 \mathrm{H}-1 \\
8 & 36-38\end{array}$ & $\begin{array}{c}832 \mathrm{~A} \\
3 \mathrm{H}-1 \\
36-38\end{array}$ & $\begin{array}{c}832 \mathrm{~A} \\
10 \mathrm{H}-1 \\
120-122\end{array}$ & $\begin{array}{c}832 \mathrm{~A} \\
20 \mathrm{H}-1 \\
280-82\end{array}$ & $\begin{array}{l}832 \mathrm{~A} \\
120 \mathrm{H}-1 \\
2 \\
20-82\end{array}$ & $\begin{array}{cc}832 \mathrm{~A} \\
1 & 20 \mathrm{H}-1 \\
2 & 80-82\end{array}$ & $\begin{array}{l}833 \mathrm{~A} \\
1 \mathrm{H}-6 \\
72-74\end{array}$ & $\begin{array}{r}833 \mathrm{~A} \\
1 \mathrm{H}-6 \\
72-74\end{array}$ & $\begin{array}{r}833 \mathrm{~A} \\
1 \mathrm{H}-6 \\
72-74\end{array}$ \\
\hline $\mathrm{SiC}$ & 48 & 48.07 & 51.8 & 58.43 & 22 & 47.71 & 45.18 & 53.33 & $52.04=5$ & 53.86 & 48.91 & 51.89 & 47.3 & 5175 & 56.04 & 55,74 & 48.23 & 45.74 & 47.43 & 52.49 & $51.84 \quad 4$ & 48.78 & 53,36 & 53 \\
\hline $\mathrm{Al}_{2} \mathrm{O}_{3}$ & $\begin{array}{l}40.48 \\
32.18\end{array}$ & 32.18 & 30.19 & 25.55 & 25.24 & 32.39 & 34.37 & 28.07 & 29.27 & 27.37 & 31.61 & 29.37 & 32.35 & 29.26 & 27.43 & 27.43 & 31.85 & $\begin{array}{l}43.74 \\
32.7\end{array}$ & 31.57 & 27.87 & 29.093 & $\begin{array}{l}30.94 \\
30.94\end{array}$ & $\begin{array}{r}27.03 \\
27.03\end{array}$ & 27.36 \\
\hline $\mathrm{MgO}$ & 0.05 & 0.12 & 0.06 & 0.04 & 0.04 & 0.11 & 0.06 & 0.15 & 0.03 & 0.16 & 0.09 & 0 & 0 & 0 & 0 & 0 & 0 & 0.08 & 0 & 0 & 0 & 0 & 0 & 0 \\
\hline $\mathrm{CaO}$ & $\begin{array}{l}16.37 \\
\end{array}$ & 17.07 & 13.64 & 9.01 & 9.44 & 16.82 & 18.89 & 12.12 & ו & 11.92 & 15.66 & 13.44 & 17.68 & 13.71 & 10.54 & 10.21 & 16.46 & 17.68 & 17.06 & 12.81 & $13.64 \quad$ & 15.99 & 12.14 & 12.45 \\
\hline $\mathrm{Na}_{2} \mathrm{O}$ & 2.34 & 1.91 & 3.67 & 6.28 & 5.85 & 2 & 1.1 & 4.8 & 4.31 & 4.62 & 2.56 & 3.83 & 1.95 & 3.77 & 5.23 & 5.22 & 2.26 & 1.33 & 1.98 & 4.3 & 3.81 & 2.61 & 4.87 & 4.25 \\
\hline $\mathrm{K}_{2} \mathrm{O}$ & 0.04 & 0.09 & 0.22 & 0.47 & 0.44 & 0.09 & 0.04 & 0.16 & 0.15 & 0.35 & 0.1 & 0.38 & 0.03 & 0.25 & 0.5 & 0.67 & 0.23 & 0.07 & 0.14 & 0.4 & 0.47 & 0.18 & 0.59 & 0.78 \\
\hline Total & 100.491 & 100.881 & 100.56 & 100.41 & 98.911 & 100.14 & 100.47 & 99.98 & $99.58 \quad 9$ & $99.8 \quad 1$ & 100.151 & 100.221 & 100.34 & 99.781 & 100.42 & $99.81 \quad$ & 100.25 & 98.73 & 99.27 & 99.551 & 100.09 & 99.74 & 99.08 & 98.92 \\
\hline A & 2 & 16.75 & 32.32 & 54.3 & 51.53 & 17 & 9.51 & 41. & 36 & 40. & 22 & 33. & 16 & 32 & 45. & 46. & 19. & 11. & 17.22 & 36.9 & 32 & 22. & 40.67 & 36. \\
\hline Or & 0.23 & 0.5 & 1.31 & 2.67 & 2.53 & 0.52 & 0.22 & 0.89 & 0.84 & 1.99 & 0.58 & 2.19 & 0.18 & 1.46 & 2.87 & 3.93 & 1.33 & 0.41 & 0.81 & 2.27 & 2.64 & 1.04 & 3.23 & 4.44 \\
\hline An & 79.28 & 82.74 & 66.37 & 43.03 & 45.93 & 81.88 & 90.27 & 57.75 & 62.395 & 57.64 & 76.73 & 64.55 & 83.22 & 65.79 & 51.18 & 49.9 & 79.01 & 87.65 & 81.97 & 60.79 & 64.66 & 76.4 & 56.09 & 59.09 \\
\hline
\end{tabular}

Table 2. Representative microprobe analyses in weight percent ( $\mathrm{wt} \%$ ) and atomic proportions of pyroxenes from Leg 134 ashes. $\mathrm{Fe}^{3+} / \mathrm{Fe}^{2+}$ calculated on the basis of charge balancing using Cameca proprietary software.

\begin{tabular}{|c|c|c|c|c|c|c|c|c|c|c|c|c|c|c|c|c|c|c|c|c|c|c|c|c|c|}
\hline Hole: & $828 \mathrm{~A}$ & $828 \mathrm{~A}$ & $828 \mathrm{~A}$ & $828 \mathrm{~A}$ & $828 \mathrm{~A}$ & $830 \mathrm{~A}$ & $832 \mathrm{~A}$ & $832 \mathrm{~A}$ & $832 \mathrm{~A}$ & $832 \mathrm{~A}$ & $832 \mathrm{~A}$ & $832 \mathrm{~A}$ & $832 \mathrm{~A}$ & $832 \mathrm{~A}$ & $832 \mathrm{~A}$ & $832 \mathrm{~A}$ & $832 \mathrm{~A}$ & $832 \mathrm{~A}$ & $832 \mathrm{~A}$ & $833 \mathrm{~A}$ & $833 \mathrm{~A}$ & $833 \mathrm{~A}$ & $\begin{array}{l}833 \mathrm{~A} \\
1 \mathrm{H}-6\end{array}$ & $833 \mathrm{~A}$ & $833 \mathrm{~A}$ \\
\hline Core, section: & $2 \mathrm{H}-6$ & $2 \mathrm{H}-6$ & $2 \mathrm{H}-6$ & $4 \mathrm{H}-2$ & $4 \mathrm{H}-2$ & $1 \mathrm{H}-3$ & $1 \mathrm{H}-4$ & $1 \mathrm{H}-4$ & $1 \mathrm{H}-4$ & 1H-4 & $3 \mathrm{H}-1$ & $3 \mathrm{H}-1$ & $10 \mathrm{H}-1$ & $10 \mathrm{H}-1$ & $10 \mathrm{H}-1$ & $20 \mathrm{H}-1$ & $20 \mathrm{H}-1$ & $20 \mathrm{H}-1$ & $20 \mathrm{H}-1$ & 1H-6 & $1 \mathrm{H}-6$ & $1 \mathrm{H}-6$ & $1 \mathrm{H}-6$ & $1 \mathrm{H}-6$ & IH-6 \\
\hline Interval $(\mathrm{cm})$ : & $7-9$ & $7-9$ & $7-9$ & $55-57$ & $55-57$ & $40-42$ & $54-56$ & $54-56$ & $54-56$ & $54-56$ & $36-38$ & 36-38 & $120-122$ & $120-122$ & $120-122$ & $280-82$ & $80-82$ & $80-82$ & $80-82$ & $72-74$ & $72-74$ & $72-74$ & $72-74$ & $72-74$ & $72-74$ \\
\hline Crystal type: & & stallite & $\begin{array}{c}\text { core of } \\
\text { grain }\end{array}$ & $\begin{array}{l}\text { large } \\
\text { cpx }\end{array}$ & $\begin{array}{l}\text { large } \\
\text { cpx }\end{array}$ & $\begin{array}{c}\text { opx } \\
\text { xenocryst }\end{array}$ & $\begin{array}{c}\text { small } \\
\mathrm{cpx}\end{array}$ & $\begin{array}{c}\text { cpx } \\
\text { grain }\end{array}$ & & $\begin{array}{c}\text { opx } \\
\text { crystallite }\end{array}$ & & $\begin{array}{c}\text { large } \\
\text { cpx }\end{array}$ & $\begin{array}{l}\text { cpx } \\
\text { grain }\end{array}$ & $\begin{array}{l}\text { large } \\
\text { cpx }\end{array}$ & $\begin{array}{l}\text { cpx } \\
\text { grain }\end{array}$ & $\begin{array}{c}\text { large } \\
\mathrm{cpx}\end{array}$ & $\begin{array}{l}\text { cpx } \\
\text { grain }\end{array}$ & $\begin{array}{l}\text { large } \\
\text { cpx }\end{array}$ & $\begin{array}{l}\text { cpx } \\
\text { grain }\end{array}$ & $\begin{array}{l}\text { cpx } \\
\text { grain }\end{array}$ & $\begin{array}{l}\text { cpx } \\
\text { grain }\end{array}$ & $\begin{array}{l}\text { cpx } \\
\text { grain }\end{array}$ & $\begin{array}{l}\text { small } \\
\text { cpx }\end{array}$ & $\begin{array}{l}\text { cpx } \\
\text { grain }\end{array}$ & $\begin{array}{l}\text { cpx in } \\
\text { glass }\end{array}$ \\
\hline $\mathrm{SiO}_{2}$ & 50.05 & 44.09 & 43.75 & 50.994 & 49.40 & 53.13 & 50.89 & 51.27 & 51.19 & 53.714 & 49.06 & 51.07 & 51.99 & 48.47 & 49.54 & $49.03 \quad 4$ & 49.86 & 50.14 & 50.92 & 48.91 & 48.50 & 49.87 & 49.70 & 49.94 & 50.38 \\
\hline $\mathrm{TiO}$ & 0.65 & 1.26 & 1.14 & 0.62 & 0.65 & 0.21 & 0.60 & & & 0.31 & 0.76 & 0.53 & 0.2 & 0.61 & 0.70 & 0.58 & 0.54 & 0.41 & 0.41 & 0.68 & 0.77 & 0.64 & 0.60 & 0.55 & 0.65 \\
\hline $\mathrm{Al}_{2} \mathrm{O}_{3}$ & 4.06 & 10.28 & 10.74 & 2.67 & 4.30 & 0.65 & 2.40 & 2.32 & 2.82 & 1.96 & 4.5 & 2.5 & 2.0 & 4.6 & 3.46 & 4.44 & 4. & 3.7 & 3. & 4.63 & 5.32 & 3.60 & 4.67 & 4.32 & 3.28 \\
\hline $\mathrm{Cr}_{2} \mathrm{O}_{3}$ & 0.14 & 0.02 & 0.01 & 0.04 & 0.03 & 0.00 & 0.00 & 0.01 & 0. & 0.11 & 0.03 & 0.0 & 0. & 0.05 & 0.00 & 0.15 & 0.12 & 0.82 & 0.37 & 0.05 & 0.11 & 0.09 & 0.06 & 0.17 & 0.01 \\
\hline $\mathrm{Fe}_{2} \mathrm{O}_{3}$ & 3.72 & 5.66 & 7.15 & 2.87 & 4.24 & 0.00 & 1.93 & 2.14 & 2.44 & 0.15 & 4.13 & 2.71 & 2.91 & 5.29 & 4.10 & 5.08 & 4.08 & 4.08 & 3.41 & 5.45 & 4.74 & 4.32 & 4.84 & 3.79 & 3.50 \\
\hline $\mathrm{MgO}$ & 15.10 & 11.71 & 11.40 & 15.57 & 14.30 & 21.13 & 14.51 & 15.57 & 14.81 & $25.42 \quad 1$ & 13.72 & 14.37 & 16.07 & 13.80 & 13.48 & $14.70 \quad 1$ & 14.58 & 15.49 & 14.88 & 13.88 & 13.47 & 13.89 & 14.25 & 14.24 & 13.98 \\
\hline & 23.06 & 20.19 & 20.69 & 19.562 & 22.01 & 1.94 & 18.92 & 17.38 & 20.36 & 2.252 & 22.12 & 21.10 & 23.88 & 22.46 & 21.95 & 22.47 & 22.98 & 22.90 & 23.33 & 22.64 & 22.62 & 22.00 & 22.38 & 22.64 & 22.33 \\
\hline & & & & & & & & & & & 0.32 & & & 0. & 0.24 & 0.25 & 0.12 & 0.10 & 0.12 & 0.16 & 0.11 & 0.37 & 0.22 & 0.10 & 0.25 \\
\hline & & & & & & & & & & & 4.7 & & & & & 2.60 & & & & & 4. & 4.94 & 3.87 & 3.78 & 5.20 \\
\hline & 0 . & & 0 & & 0 & 0 & 0.32 & & & 0 & 0.32 & 0. & & 0. & 0.40 & 0.28 & 0.28 & 0.27 & 0.33 & 0.36 & 0.30 & 0.42 & 0.39 & 0.42 & 0.40 \\
\hline Total & 99.96 & 99.35 & 100.39 & 100.14 & 99.93 & 100. & 99.36 I & 100 & 100 & 100 & 99.71 & 100. & 99.8 & 99.45 & 99.48 & 99.58 & 99.99 & $100.01 \mathrm{I}$ & 100.6 & 100.281 & 100.131 & 100.141 & 100.98 & 99.95 & 99.97 \\
\hline $\mathrm{Si}$ & 1.841 & 1.655 & 1.630 & 1.886 & 1.831 & 1.982 & 1.914 & 1.908 & 1. & 1.947 & 1.833 & 1.8 & & 1.815 & 1.861 & 1.823 & 1.846 & 1.848 & 1.869 & 1.816 & 1.805 & 1.857 & 1.828 & 1.850 & 1.875 \\
\hline $\mathrm{Ti}$ & & & 0. & 8 & 0.0 & & 0.017 & 0.0 & & & 0.021 & & & 0.017 & 220 & 0.016 & 0.015 & 0.012 & 0.011 & 0.019 & 0.022 & 0.018 & 0.017 & 0.015 & 0.018 \\
\hline Al & 82 & & 0. & & 0.1 & & 0.106 & 0.1 & & 0. & 0.199 & & & & 0.151 & 0.195 & 0.182 & 0.164 & 0.159 & 0.203 & 0.233 & 0.158 & 0.203 & 0.189 & 0.144 \\
\hline $\mathrm{Cr}$ & 0.004 & & 0. & & 0.0 & & 0.000 & 0.0 & & 0. & 0.001 & 0. & & 0.0 & 0. & 0.0 & 0.0 & 0.024 & 0.0 & 0.002 & 0.003 & 0.003 & 0.002 & 0.005 & 0.000 \\
\hline $\mathrm{Fe}^{3+}$ & & & & & 0.1 & & 0.055 & 0.0 & & & 0.1 & 0. & & & 16 & 0.142 & 0.113 & 0.113 & 0.0 & 0. & 0.1 & 0.121 & 0.134 & 0. & 0.0 \\
\hline $\mathrm{M}$ & & & 0.639 & & 0.798 & & 0.814 & & & & 0.764 & 0.7 & & & 0.754 & 0.815 & 0.804 & 0.851 & 0.8 & & & 0.771 & & & 0.7 \\
\hline C & 0 & 0. & 0. & 0. & 0.87 & & 0.763 & 0.6 & & & 0.886 & 0.8 & & 0.5 & 0.883 & 0.895 & 0.911 & 0.904 & 0.9 & & & & & & 0.891 \\
\hline$M$ & & & 0 & 0. & 0.0 & 0 & 0.018 & 0. & & 0. & 0.010 & 0.0 & 0. & 0. & 0.008 & 0.008 & 0.004 & 0.003 & 0.0 & & 0.004 & 0.012 & & 0. & 0.008 \\
\hline $\mathrm{Fe}$ & & & & 0 & 0. & 0 & 0. & 0 & & & 0.1 & 0. & & 0.1 & 0.177 & 0.081 & 0.101 & 0.062 & 0.098 & 0.109 & 0.130 & 0.154 & 0.119 & 0.117 & 0.162 \\
\hline $\mathrm{N}:$ & & & & & & & & & & 1 & 0.023 & 31 & 3 & 0.020 & 0.029 & 0.020 & 0.020 & 0.020 & 0.024 & 0.026 & 0.022 & 0.031 & 0.028 & 0.030 & 0.029 \\
\hline Wo & 46 & 44. & 45 & 39.52 & 45 . & 3 & 3 & 35 & & 4.47 & 46.0 & 43. & 47. & 46.43 & 45.59 & 46.12 & 47.13 & 46.78 & 47.60 & 46.53 & 47.08 & 45.36 & 45.86 & 47.03 & 46.05 \\
\hline En & 43 & 36. & & 44.25 & & 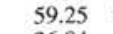 & 41 & & & 701 & 39. & & & & 38 & 41 & 41 & 44 & 42 & 39 & 38 & 39.84 & 40 & 41.15 & 40.10 \\
\hline Fs & 10.13 & 19.12 & 20.07 & 16.24 & 13.84 & 36.84 & 18.73 & 20.29 & 16.21 & 25.39 & 14.27 & 15.05 & 7.69 & 13.89 & 15.48 & 11.89 & 11.28 & 9.22 & 10.17 & 13.77 & 13.93 & 14.80 & 13.51 & 11.82 & 13.85 \\
\hline
\end{tabular}

Note: cpx: clinopyroxene; opx: orthopyroxene. 


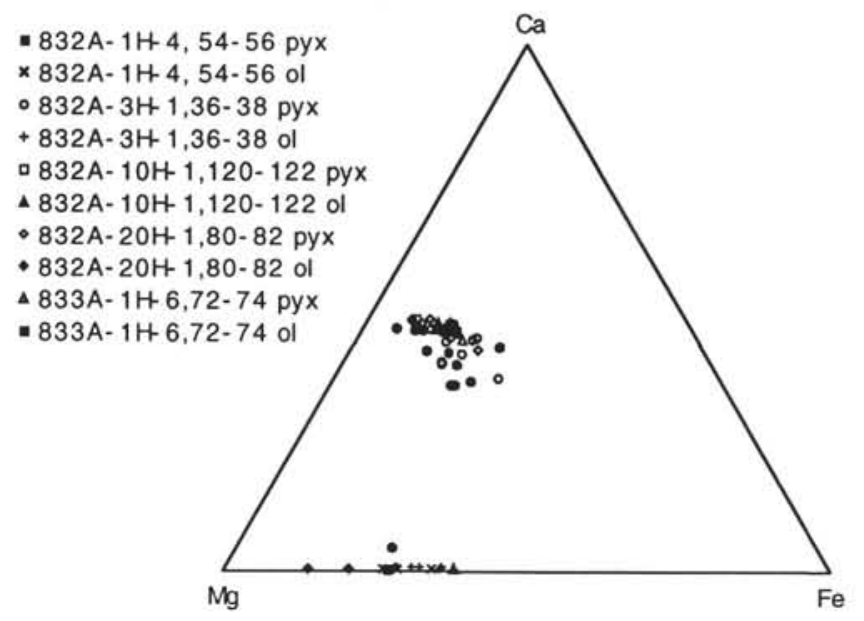

Figure 3. Projections of pyroxene and olivine compositions in terms of relative proportions of $\mathrm{Ca}, \mathrm{Mg}$, and $\mathrm{Fe}$, from North Aoba Basin Sites 832 and 833 .

$830 \mathrm{~A}-3 \mathrm{H}-4,108-110 \mathrm{~cm}$ ) and North Aoba Basin (Sample 134-832A$1 \mathrm{H}-4,54-56 \mathrm{~cm})$. Orthopyroxene $\left(\mathrm{Ca}_{5} \mathrm{Mg}_{70} \mathrm{Fe}_{25}\right)$ also occurs as small crystals in glass from the latter sample. The thermometer of Lindsley and Anderson (1983) was applied to Sample 134-832A-1H-4, 54-56 $\mathrm{cm}$, on the assumption that the two pyroxenes were in equilibrium and that an equilibration pressure of $10^{5} \mathrm{~Pa}$ is appropriate. The results indicate an equilibration temperature of $1050^{\circ}-1100^{\circ} \mathrm{C}$. The only other pyroclast containing orthopyroxene $\left(\mathrm{Ca}_{4} \mathrm{Mg}_{59} \mathrm{Fe}_{37}\right)$ is from the forearc (Sample 134-830A-1H-3, 40-42 cm) where the large size of the hypersthene in the pumice suggests it may be a xenocryst. Olivine is conspicuously more abundant in the North Aoba Basin ashes than in those of the forearc. In fact, in the latter it was found in only one instance (Sample 134-828A-2H-6, 7-9 cm), where it has the most magnesian composition (Table 3 ) encountered in this study $\left(\mathrm{FO}_{87}\right)$. The only other pyroclast with comparable olivine $\left(\mathrm{Fo}_{86}\right)$ is Sample 134-832A-20H-1, $80-82 \mathrm{~cm}$, a tuff from $146 \mathrm{mbsf}$ in the North Aoba Basin. More commonly, olivine compositions in the North Aoba Basin lie within the range $\mathrm{Fo}_{77}$ to $\mathrm{Fo}_{62}$. Assuming that most of the ashes originated from the Central Chain volcanoes, the lack of olivine at forearc sites probably means that as a higher density phase it had already settled out from the ash clouds before the fine material reached the forearc slope to the west. The only other crystalline constituents of the ashes are magnetite, titanomagnetite, apatite, and quartz.

\section{GLASS GEOCHEMISTRY}

No bulk analyses of ash layers were performed on the Leg 134 ashes since aeolian and subaqueous fractionation, together with some reworking, severely limits the value of such data. Emphasis has therefore been placed on the analysis of glasses as being the best indicators of magma compositions. They are used to distinguish magmatic affinities, to attempt correlation, and to try to establish the provenance of particular ash horizons. Glass occurs in a variety of forms from blocky particles to sheaves, vesicular scoria, cellular pumice, and cuspate shards. There is no strong correlation between form and composition, although there is a tendency for blocky fragments to have a more basic composition and for the highly cellular and thin-shard varieties to be more siliceous.

Electron microprobe analyses of the glasses are presented in Tables 4 (forearc sites) and 5 (North Aoba Basin sites). For comparative purposes the analyses have been recalculated to $100 \%$ volatile-free, but the original totals are also shown. Low totals in many of these raw analyses presumably reflect alteration or hydration during diagenesis. In some instances, however, the glass may have contained significant amounts of water at the time of its eruption. Not unexpectedly, within any given hole the degree of alteration, as expressed in the low total, increases with depth. The deepest ( $310 \mathrm{mbsf}$ ) ash or tuff analyzed (Sample 134-833A-25R-2, 29-31 cm) gave much the lowest total ( $82 \mathrm{wt} \%$ and $86 \mathrm{wt} \%$ ), and it is evident from its chemistry (e.g., anomalously low $\mathrm{Na}_{2} \mathrm{O}$ and $\mathrm{K}_{2} \mathrm{O}$ ) that the glass has undergone severe alteration.

Almost all of the ash layers studied contain glass fragments of various compositions. For example, Sample 134-832A-1H-4, 54-56 $\mathrm{cm}$, from a depth of $3 \mathrm{mbsf}$, has shards ranging in composition from $49 \mathrm{wt} \%$ to $62 \mathrm{wt} \% \mathrm{SiO}_{2}$. Sample 134-828A-2H-6, 7-9 cm, also contains a spread of compositions from $49 \mathrm{wt} \%$ to $64 \mathrm{wt} \% \mathrm{SiO}_{2}$. The ashes collected for this study were generally of the order of $1-2 \mathrm{~cm}$ in thickness and were homogeneous in aspect. In each case, a single integrated sample was taken so that there is no way of knowing whether the different glasses are uniformly distributed throughout or if they are separated in thin, but compositionally distinct laminae, indiscernible on the hand-specimen scale. However, Jezek (1976) also found a range of $\mathrm{SiO}_{2}$ concentrations in glasses from single ash layers cored in the North Fiji Basin. Eade and Gregory (1992) similarly report widely differing glass chemistry in ooze from a single horizon in the same basin. There are a number of possible explanations for this observation. In the first place, a relatively low sedimentation rate and reduced explosive volcanic activity may result in reworking and homogenization of two or more discrete horizons. This might conceivably be brought about by current action or bioturbation. Eade and Gregory (1992) conclude that the latter is an important process in ash mixing and that only those layers thicker than about $2 \mathrm{~cm}$ retain their original form. Several of the composite ash layers investigated here contain foraminifers and nannofossils (e.g., Sample 134-828A-2H-6, $7-9 \mathrm{~cm}$ ). Although this might indicate reworking, it does not necessarily demand this explanation. Several separate ash falls might be represented in what appears to be a single layer, with fossils accumulating during the intervals between eruptions. Finely divided glass

Table 3. Representative microprobe analyses in weight percent (wt \%) and atomic proportions of olivines from Leg 134 ashes. Total Fe reported as $\mathrm{FeO}$.

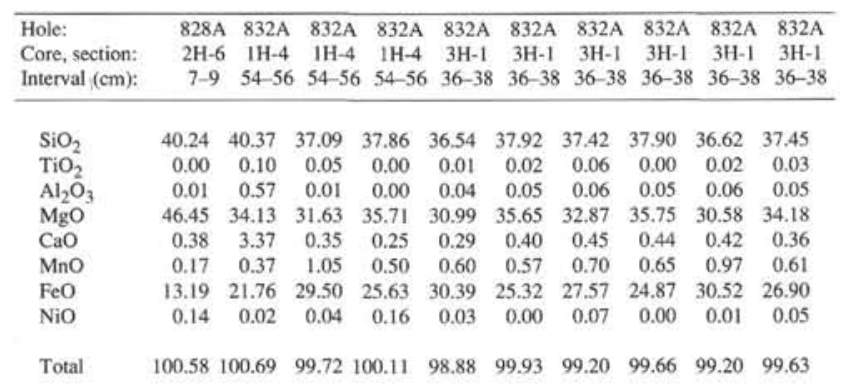

$\begin{array}{llllllllll}\text { Sum of cations } 3.003 & 2.947 & 2.992 & 2.999 & 2.996 & 2.995 & 2.988 & 2.995 & 2.993 & 2.997\end{array}$ 
Table 4. Major element compositions in weight percent (wt \%) of forearc glasses (Sites 828, 830, and 831).

\begin{tabular}{|c|c|c|c|c|c|c|c|c|c|c|c|c|c|c|c|c|c|}
\hline $\begin{array}{l}\text { Hole: } \\
\text { Core, section: } \\
\text { Interval }(\mathrm{cm}) \text { : } \\
\text { Depth (mbsf): }\end{array}$ & $\begin{array}{c}828 \mathrm{~A} \\
2 \mathrm{H}-6 \\
7-9 \\
11.88\end{array}$ & $\begin{array}{c}828 \mathrm{~A} \\
2 \mathrm{H}-6 \\
7-9 \\
11.88\end{array}$ & $\begin{array}{c}828 \mathrm{~A} \\
2 \mathrm{H}-6 \\
7-9 \\
11.88\end{array}$ & $\begin{array}{l}828 \mathrm{~A} \\
4 \mathrm{H}-2 \\
55-57 \\
25.46\end{array}$ & $\begin{array}{c}828 \mathrm{~A} \\
4 \mathrm{H}-2 \\
55-57 \\
25.46\end{array}$ & $\begin{array}{l}828 \mathrm{~A} \\
4 \mathrm{H}-2 \\
55-57 \\
25.46\end{array}$ & $\begin{array}{c}828 \mathrm{~A} \\
4 \mathrm{H}-2 \\
55-57 \\
25.46\end{array}$ & $\begin{array}{l}828 \mathrm{~A} \\
4 \mathrm{H}-2 \\
55-57 \\
25.46\end{array}$ & $\begin{array}{c}828 \mathrm{~A} \\
4 \mathrm{H}-2 \\
55-57 \\
25.46\end{array}$ & $\begin{array}{l}828 \mathrm{~A} \\
4 \mathrm{H}-2 \\
55-57 \\
25.46\end{array}$ & $\begin{array}{l}828 \mathrm{~A} \\
4 \mathrm{H}-2 \\
55-57 \\
25.46\end{array}$ & $\begin{array}{c}830 \mathrm{~A} \\
1 \mathrm{H}-3 \\
40-42 \\
3.41\end{array}$ & $\begin{array}{c}830 \mathrm{~A} \\
1 \mathrm{H}-3 \\
40-42 \\
3.41\end{array}$ & $\begin{array}{c}830 \mathrm{~A} \\
1 \mathrm{H}-3 \\
40-42 \\
3.41\end{array}$ & $\begin{array}{c}830 \mathrm{~A} \\
1 \mathrm{H}-3 \\
40-42 \\
3.41\end{array}$ & $\begin{array}{c}830 \mathrm{~A} \\
1 \mathrm{H}-3 \\
40-42 \\
3.41\end{array}$ & $\begin{array}{c}830 \mathrm{~A} \\
1 \mathrm{H}-3 \\
40-42 \\
3.41\end{array}$ \\
\hline $\begin{array}{l}\mathrm{SiO}_{2} \\
\mathrm{TiO}_{2} \\
\mathrm{Al}_{2} \mathrm{O}_{3} \\
\mathrm{FeO} \\
\mathrm{MnO} \\
\mathrm{MgO} \\
\mathrm{CaO} \\
\mathrm{Na}_{2} \mathrm{O} \\
\mathrm{K}_{2} \mathrm{O}\end{array}$ & $\begin{array}{r}50.35 \\
0.86 \\
16.01 \\
10.02 \\
0.16 \\
6.12 \\
12.09 \\
2.75 \\
1.64\end{array}$ & $\begin{array}{r}50.91 \\
0.93 \\
15.92 \\
10.03 \\
0.18 \\
5.88 \\
11.68 \\
2.79 \\
1.68\end{array}$ & $\begin{array}{r}53.50 \\
1.02 \\
16.90 \\
11.42 \\
0.31 \\
3.24 \\
7.64 \\
3.28 \\
2.70\end{array}$ & $\begin{array}{r}53.52 \\
0.89 \\
15.37 \\
11.67 \\
0.29 \\
4.51 \\
9.67 \\
2.90 \\
1.19\end{array}$ & $\begin{array}{r}53.03 \\
0.83 \\
15.02 \\
13.65 \\
0.23 \\
4.43 \\
9.20 \\
2.68 \\
0.91\end{array}$ & $\begin{array}{r}54.93 \\
1.21 \\
14.10 \\
17.13 \\
0.26 \\
2.46 \\
6.59 \\
1.52 \\
1.80\end{array}$ & $\begin{array}{r}52.47 \\
0.84 \\
15.02 \\
12.23 \\
0.14 \\
5.33 \\
10.19 \\
2.63 \\
1.14\end{array}$ & $\begin{array}{r}53.00 \\
0.98 \\
15.59 \\
11.94 \\
0.36 \\
4.62 \\
9.61 \\
2.56 \\
1.34\end{array}$ & $\begin{array}{r}53.03 \\
0.98 \\
15.36 \\
12.07 \\
0.38 \\
4.85 \\
9.74 \\
2.31 \\
1.28\end{array}$ & $\begin{array}{r}53.77 \\
0.94 \\
14.71 \\
12.44 \\
0.31 \\
4.64 \\
9.23 \\
2.64 \\
1.32\end{array}$ & $\begin{array}{r}52.84 \\
1.08 \\
14.75 \\
13.14 \\
0.22 \\
4.83 \\
9.32 \\
2.51 \\
1.30\end{array}$ & $\begin{array}{r}67.05 \\
0.56 \\
15.12 \\
5.53 \\
0.13 \\
1.10 \\
3.34 \\
3.51 \\
3.66\end{array}$ & $\begin{array}{r}66.96 \\
0.68 \\
14.77 \\
5.18 \\
0.24 \\
1.21 \\
3.21 \\
4.07 \\
3.68\end{array}$ & $\begin{array}{r}66.94 \\
0.59 \\
14.80 \\
5.32 \\
0.16 \\
1.13 \\
3.37 \\
4.00 \\
3.69\end{array}$ & $\begin{array}{r}67.93 \\
0.57 \\
15.08 \\
4.90 \\
0.13 \\
1.01 \\
3.08 \\
3.48 \\
3.81\end{array}$ & $\begin{array}{r}67.11 \\
0.63 \\
14.99 \\
5.20 \\
0.32 \\
1.11 \\
3.10 \\
3.76 \\
3.79\end{array}$ & $\begin{array}{r}67.13 \\
0.57 \\
14.99 \\
4.97 \\
0.21 \\
1.11 \\
3.29 \\
3.96 \\
3.76\end{array}$ \\
\hline Original Total & 99.36 & 98.62 & 94.85 & 98.21 & 99.52 & 97.88 & 99.64 & 99.10 & 98.91 & 99.02 & 98.71 & 100.20 & 99.86 & 99.30 & 98.95 & 100.31 & 99.47 \\
\hline
\end{tabular}

Table 4 (continued).

\begin{tabular}{|c|c|c|c|c|c|c|c|c|c|c|c|c|c|c|c|c|c|}
\hline $\begin{array}{l}\text { Hole: } \\
\text { Core, section: } \\
\text { Interval }(\mathrm{cm}) \text { : } \\
\text { Depth (mbsf): }\end{array}$ & $\begin{array}{c}830 \mathrm{~A} \\
3 \mathrm{H}-3 \\
75-76 \\
20.26\end{array}$ & $\begin{array}{l}830 \mathrm{~A} \\
3 \mathrm{H}-3 \\
75-76 \\
20.26\end{array}$ & $\begin{array}{c}830 \mathrm{~A} \\
3 \mathrm{H}-3 \\
75-76 \\
20.26\end{array}$ & $\begin{array}{l}830 \mathrm{~A} \\
3 \mathrm{H}-3 \\
75-76 \\
20.26\end{array}$ & $\begin{array}{l}830 \mathrm{~A} \\
3 \mathrm{H}-3 \\
75-76 \\
20.26\end{array}$ & $\begin{array}{l}830 \mathrm{~A} \\
3 \mathrm{H}-3 \\
75-76 \\
20.26\end{array}$ & $\begin{array}{l}830 \mathrm{~A} \\
3 \mathrm{H}-3 \\
75-76 \\
20.26\end{array}$ & $\begin{array}{l}830 \mathrm{~A} \\
3 \mathrm{H}-3 \\
75-76 \\
20.26\end{array}$ & $\begin{array}{l}831 \mathrm{~A} \\
3 \mathrm{H}-6 \\
54-55 \\
14.45\end{array}$ & $\begin{array}{l}831 \mathrm{~A} \\
3 \mathrm{H}-6 \\
54-55 \\
14.45\end{array}$ & $\begin{array}{l}831 \mathrm{~A} \\
3 \mathrm{H}-6 \\
54-55 \\
14.45\end{array}$ & $\begin{array}{l}831 \mathrm{~A} \\
3 \mathrm{H}-6 \\
54-55 \\
14.45\end{array}$ & $\begin{array}{l}831 \mathrm{~A} \\
3 \mathrm{H}-6 \\
54-55 \\
14.45\end{array}$ & $\begin{array}{l}831 \mathrm{~A} \\
3 \mathrm{H}-6 \\
54-55 \\
14.45\end{array}$ & $\begin{array}{l}831 \mathrm{~A} \\
3 \mathrm{H}-6 \\
54-55 \\
14.45\end{array}$ & $\begin{array}{l}831 \mathrm{~A} \\
3 \mathrm{H}-6 \\
54-55 \\
14.45\end{array}$ & $\begin{array}{l}831 \mathrm{~A} \\
3 \mathrm{H}-6 \\
54-55 \\
14.45\end{array}$ \\
\hline $\begin{array}{l}\mathrm{SiO}_{2} \\
\mathrm{TiO}_{2} \\
\mathrm{Al}_{2} \mathrm{O}_{3} \\
\mathrm{FeO} \\
\mathrm{MnO} \\
\mathrm{MgO} \\
\mathrm{CaO} \\
\mathrm{Na}_{2} \mathrm{O} \\
\mathrm{K}_{2} \mathrm{O}\end{array}$ & $\begin{array}{r}70.80 \\
0.63 \\
15.60 \\
3.75 \\
0.13 \\
0.84 \\
2.52 \\
2.74 \\
3.00\end{array}$ & $\begin{array}{r}69.90 \\
0.58 \\
15.05 \\
3.34 \\
0.20 \\
0.76 \\
2.35 \\
3.41 \\
4.39\end{array}$ & $\begin{array}{r}70.35 \\
0.48 \\
15.55 \\
2.67 \\
0.07 \\
0.70 \\
2.26 \\
3.33 \\
4.59\end{array}$ & $\begin{array}{r}70.01 \\
0.57 \\
15.13 \\
2.97 \\
0.13 \\
0.75 \\
2.35 \\
3.75 \\
4.33\end{array}$ & $\begin{array}{r}70.29 \\
0.53 \\
15.29 \\
3.03 \\
0.07 \\
0.72 \\
2.25 \\
3.39 \\
4.43\end{array}$ & $\begin{array}{r}69.78 \\
0.61 \\
15.09 \\
3.28 \\
0.06 \\
0.82 \\
2.22 \\
3.61 \\
4.55\end{array}$ & $\begin{array}{r}69.97 \\
0.64 \\
15.09 \\
2.89 \\
0.09 \\
0.80 \\
2.35 \\
3.59 \\
4.58\end{array}$ & $\begin{array}{r}69.34 \\
0.54 \\
15.51 \\
3.22 \\
0.14 \\
0.85 \\
2.42 \\
3.71 \\
4.27\end{array}$ & $\begin{array}{r}52.89 \\
0.92 \\
14.53 \\
11.88 \\
0.20 \\
5.61 \\
10.27 \\
2.59 \\
1.12\end{array}$ & $\begin{array}{r}55.96 \\
0.95 \\
15.91 \\
10.80 \\
0.35 \\
3.47 \\
7.81 \\
3.14 \\
1.60\end{array}$ & $\begin{array}{r}56.36 \\
0.83 \\
15.49 \\
11.13 \\
0.16 \\
3.37 \\
7.51 \\
3.56 \\
1.59\end{array}$ & $\begin{array}{r}56.20 \\
0.90 \\
15.78 \\
10.66 \\
0.20 \\
3.32 \\
7.78 \\
3.55 \\
1.59\end{array}$ & $\begin{array}{r}53.53 \\
0.96 \\
14.76 \\
12.30 \\
0.26 \\
4.43 \\
9.43 \\
2.94 \\
1.39\end{array}$ & $\begin{array}{r}53.47 \\
0.91 \\
14.87 \\
12.42 \\
0.14 \\
4.49 \\
9.51 \\
2.92 \\
1.26\end{array}$ & $\begin{array}{r}56.09 \\
0.98 \\
15.12 \\
11.34 \\
0.22 \\
3.54 \\
7.71 \\
3.52 \\
1.48\end{array}$ & $\begin{array}{r}56.14 \\
1.00 \\
15.33 \\
11.31 \\
0.22 \\
3.38 \\
7.45 \\
3.55 \\
1.62\end{array}$ & $\begin{array}{r}57.17 \\
1.19 \\
13.52 \\
13.33 \\
0.42 \\
3.18 \\
6.86 \\
2.46 \\
1.86\end{array}$ \\
\hline Original Total & 95.33 & 95.78 & 95.67 & 96.47 & 95.27 & 95.82 & 95.70 & 97.43 & 99.55 & 98.79 & 98.40 & 97.81 & 98.38 & 98.38 & 98.40 & 100.06 & 97.20 \\
\hline
\end{tabular}

Notes: For comparative purposes analyses have been recalculated to $100 \%$. "Original total" indicates the raw total returned by electron microprobe analysis. Total Fe is reported as $\mathrm{FeO}$. Columns showing the same sample number refer to analyses of different glass shards in the same ash specimen.

may also remain in suspension for a considerable time and simply incorporate forams and nannofossils as it settles. Sometimes (e.g., Sample 134-831A-3H-6, 54-55 cm, from Bougainville Guyot) the glass is restricted to two fairly well-defined compositional groups. Again, this might be accounted for by a low background sedimentation rate and subsequent mixing as outlined previously. However, it is just as conceivable that two separate eruptions, discharging material of different compositions, occurred more or less simultaneously. For instance, Warden (1967) records the simultaneous eruption of hypersthene andesite and olivine basalt from Lopevi volcano.

Another possibility is that the composite ash layer incorporates glasses of differing composition derived from a layered magma chamber. If the eruptions occurred over a period of months, or even a few years, the rate of ash fall would exceed the background sedimentation rate and the layer would include glasses of different composition. It is not uncommon for significant compositional changes to occur on this sort of timescale during a succession of explosive phases. An example is the 1902-1903 eruption of the Soufriere Volcano, St. Vincent (Robson and Tomblin, 1966). The more evolved magma from the upper part of the conduit or chamber is erupted first and is followed by more basic compositions as magma from lower in the system is tapped.

A plot of $\mathrm{K}_{2} \mathrm{O}$ vs. $\mathrm{SiO}_{2}$ (Fig. 4) for all glasses analyzed in this study matches the high-K and low-K trends identified previously in the New Hebrides volcanic rocks (e.g., Mallick, 1973; Gorton, 1977). In the recently active Central Chain volcanoes (i.e., Ambrym, Aoba, and Santa Maria; Fig. 1), from which the Leg 134 ashes are most likely to have been derived, fairly distinctive trends have been recognized for individual volcanoes or groups of volcanoes (Macfarlane et al.,1988). The latter authors attribute the two trends to the influence of the subducting d'Entrecasteaux Zone (DEZ) in modifying the thermal environment of the source region. Low-K lavas are generated by high degrees of partial melting in the mantle. The subducting DEZ reduces temperatures, forces magma genesis to deeper levels, and results in

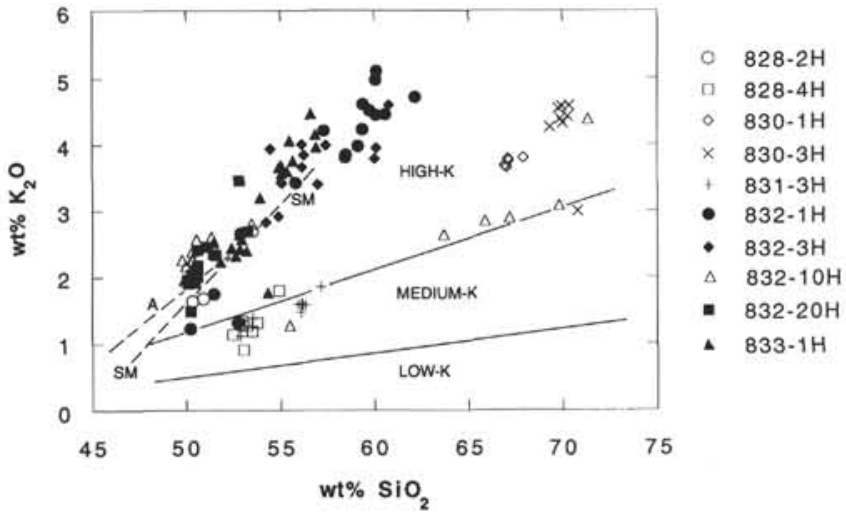

Figure 4. Variation of weight percent $\mathrm{K}_{2} \mathrm{O}$ vs. $\mathrm{SiO}_{2}$ for glasses from Leg 134 ashes. Sample numbers are abbreviated in legend; details are as follows: 828A-2H-6, 7-9 cm; 828A-4H-2, 55-57 cm; 830A-1H-3, 40-42 cm; 830A$3 \mathrm{H}-3,75-76 \mathrm{~cm} ; 831 \mathrm{~A}-3 \mathrm{H}-6,54-55 \mathrm{~cm} ; 832 \mathrm{~A}-1 \mathrm{H}-4,54-56 \mathrm{~cm} ; 832 \mathrm{~A}-3 \mathrm{H}-1$, $36-38 \mathrm{~cm} ; 832 \mathrm{~A}-10 \mathrm{H}-1,120-122 \mathrm{~cm} ; 832 \mathrm{~A}-20 \mathrm{H}-1,80-82 \mathrm{~cm} ; 833-1 \mathrm{H}-6$, $72-74 \mathrm{~cm}$. Solid lines dividing low- $\mathrm{K}$, medium- $\mathrm{K}$, and high- $\mathrm{K}$ fields are from Macfarlane et al. (1988), as are the dashed lines representing trends for A (=Aoba) and SM (=Santa Maria).

lower degrees of partial melting and thus the high-K series. Because of the collision vector (see Collot et al., this volume; Greene et al., this volume) the influence of the DEZ moves northwards along the arc with time and there is a reversion to low- $\mathrm{K}$ lavas in its wake.

The separation into two trends seen in the $\mathrm{K}_{2} \mathrm{O}$ vs. $\mathrm{SiO}_{2}$ plot (Fig. 4) is also apparent in the plot of $\mathrm{K}_{2} \mathrm{O}$ vs. $\mathrm{MgO}$ (Fig. 5), but cannot be reproduced in bivariant plots of any other pairs of major oxides. Some such plots (e.g., $\mathrm{CaO}$ vs. $\mathrm{MgO}$; Fig. 6) produce a single linear trend 


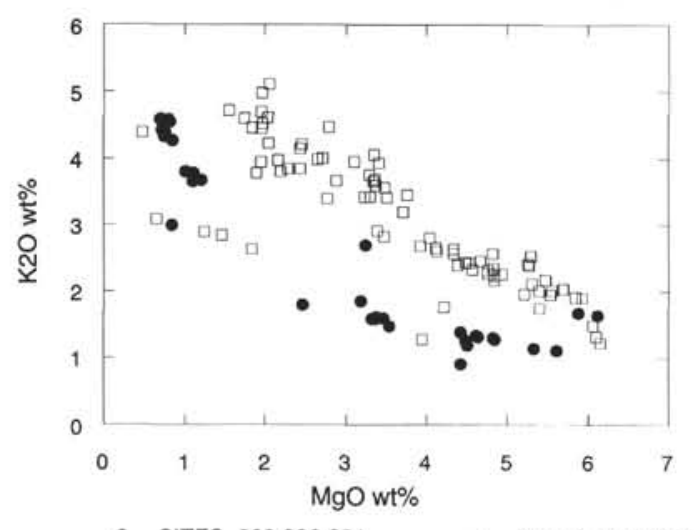

- SITES $828,830,831$

ㅁ SITES 8328833

Figure 5. Variation of weight percent $\mathrm{MgO}$ vs. $\mathrm{K}_{2} \mathrm{O}$ for glasses from Leg 134 ashes. Samples from forearc Sites 828, 830, and 831 are distinguished from those of the North Aoba Basin, Sites 832 and 833 .

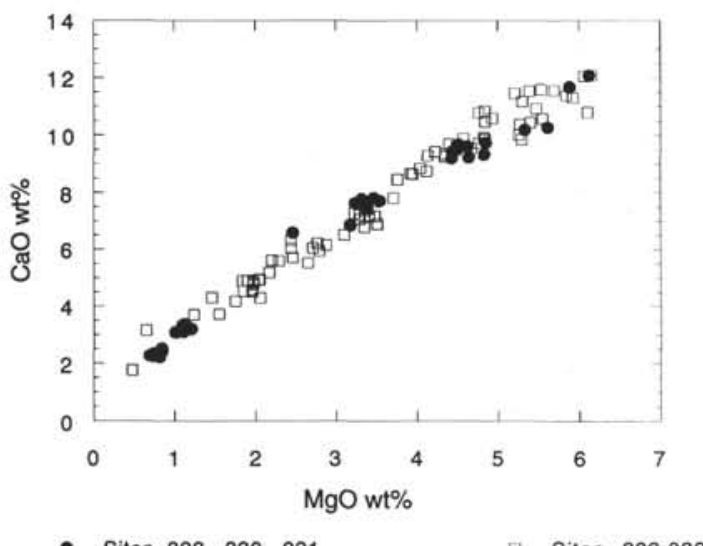

Figure 6. Variation of weight percent $\mathrm{CaO}$ vs. $\mathrm{MgO}$ for glasses from $\mathrm{Leg} 134$ ashes. Samples from forearc Sites 828, 830, and 831 are distinguished from those of the North Aoba Basin, Sites 832 and 833.

but most result in a broad scatter. In a plot of $\mathrm{FeO} / \mathrm{MgO}$ vs. $\mathrm{SiO}_{2}$ (Fig. 7), most of the glass compositions fall in the tholeiitic field of Miyashiro (1974) with a small group of rhyolitic glasses from Site 830 plotting on the calc-alkaline side of the divide.

It is evident from the $\mathrm{K}_{2} \mathrm{O}$ vs. $\mathrm{SiO}_{2}$ plot of Figure 4 that the high-K trend is abundantly represented by glasses from all of the North Aoba Basin sites. However, this trend is poorly represented at the forearc sites and occurs only in Sample 134-828A-2H-6, 7-9 cm, from the NDR. An interpretation is that eruptions of high-K magma from the Central Chain volcanoes generally fail to reach the forearc sites owing to the prevailing westerly wind in the altitude $2-18 \mathrm{~km}$ (Eade and Gregory, 1992). Conversely the low-K series tends to be rather weakly represented in the North Aoba Basin ashes (apart from Sample 832A$10 \mathrm{H}-1,120-122 \mathrm{~cm}$ ), but is quite prominently represented in the forearc sites. For example, the Bougainville Guyot specimen (Sample 134-831A-3H-6, 54-55 cm) and also one from the NDR (Sample $134-828 \mathrm{~A}-4 \mathrm{~h}-2,55-57 \mathrm{~cm}$ ) contain glasses belonging exclusively to the low-K trend. The implication is that, for whatever reason, the low-K glasses were more readily distributed across the forearc sites. Perhaps the explanation is that the high- $\mathrm{K}$ ashes were the product of more volatile-rich magmas and more explosive eruptions. High velocity eruption columns carried the ash rapidly to high altitude where distribution was controlled by westerly winds. In contrast, the distribution of low-K ashes, from low volatile less explosive eruptions, was governed mainly by the low level southeast trade winds. Despite some

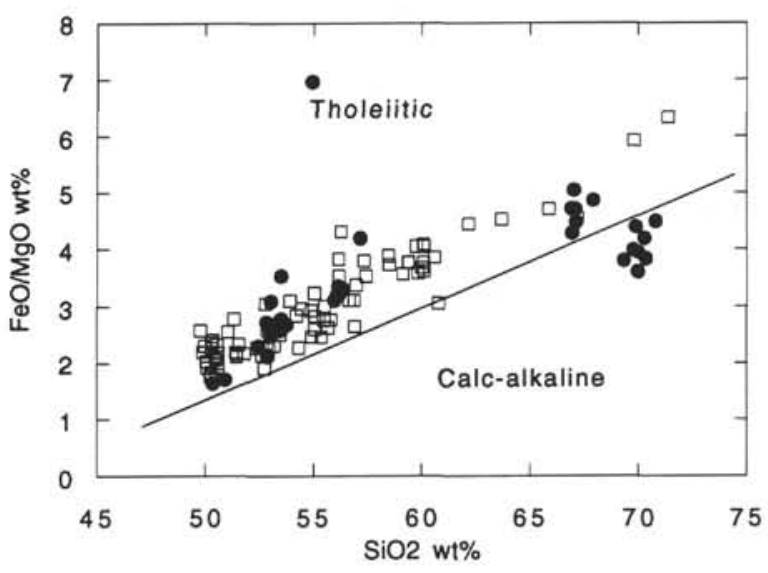

- Sites $828,830,831$

- Sites 832,833

Figure 7. Variation of $\mathrm{FeO}$ (total $\mathrm{Fe}$ as $\mathrm{FeO}$ )/ $\mathrm{MgO}$ vs. $\mathrm{SiO}_{2}$ for glasses from Leg 134 ashes. Samples from forearc Sites 828,830, and 831 are distinguished from those of the North Aoba Basin, Sites 832 and 833. Discriminant line between tholeiitic and calc-alkaline fields is from Miyashiro (1974).

overlap, it must be concluded that two different groups of volcanoes or episodes of eruption are represented, one providing the dominant input into the North Aoba Basin sites and the other to the forearc sites.

\section{PROVENANCE OF ASHES}

Apart from the generalized distinctions made above, the following more specific categories or groups may be recognized:

1. A high-silica rhyodacite ash $\left(69 \mathrm{wt} \% \mathrm{SiO}_{2}\right)$ forms a wellmarked layer at Site 830 (Sample 134-830A-3H-3, 75-76 cm) and an overlying pumice fragment (Sample $134-830 \mathrm{~A}-1 \mathrm{H}-3,40-42 \mathrm{~cm}$ ) is of similar composition. The $\mathrm{SiO}_{2}$ content remains almost constant in the group of glass fragments analyzed but there is some variation in $\mathrm{K}_{2} \mathrm{O}$, which might be due to either analytical error or variable leaching of potassium. These ash samples lie in an intermediate position between the two main trends and are referred to a medium-K suite (e.g., Macfarlane et al. 1988, their Fig. 19). Glass of similar composition is also found in one of the North Aoba Basin sites (Sample 134-832A-10H-1, 120-122 cm). However, it seems likely that Site 830 is relatively close to and downwind from the source of this ash. It is quite likely that these rhyodacite glass samples equate with the whole-rock dacite samples of medium-K type described by Crawford et al. (1988) from the submarine volcanoes of Epia and Epib (Fig. 1). Extensive subaerial deposits of such pumice are also found on Epi, Tongoa, and Tongariki (Warden, 1967) and are thought to have been emitted during paroxysmal eruptions associated with caldera development in the Epi-Tongariki group of islands. Orthopyroxene in the pumice from Site 830 (Sample 134-830A-1H-3, 40-42 cm) is almost identical in composition to that reported in dacite pumice from Epib submarine volcano (Crawford et al., 1988).

The uppermost pumice layer on the islands is thought to have been erupted between 400 and 650 yr ago (Warden, 1967), and Crawford et al. (1988) suggest major eruptions between 3000 and $4000 \mathrm{yr}$ ago. Explosive eruptions associated with caldera subsidence are likely to have been relatively high magnitude events. Most of the material would have been transported in the upper westerly winds, thus largely avoiding the North Aoba Basin. However, it would seem that a certain amount of material was carried $150 \mathrm{~km}$ or so by the low-level southeast trade winds to Site 830 .

2. One glass composition ( $53 \mathrm{wt} \% \mathrm{SiO}_{2}$ and $1 \mathrm{wt} \% \mathrm{~K}_{2} \mathrm{O}$ ) on the low- $\mathrm{K}$ trend is strongly represented at sites in the forearc, on Bougainville Guyot (Sample 134-831A-3H-6, 54-55 cm) and on the NDR (Sam- 
P.E. BAKER ET AL.

Table 5. Major element compositions (wt \%) of North Aoba Basin glasses (Sites 832 and 833).

\begin{tabular}{|c|c|c|c|c|c|c|c|c|c|c|c|c|c|}
\hline $\begin{array}{l}\text { Hole: } \\
\text { Core, section: } \\
\text { Interval }(\mathrm{cm}) \text { : } \\
\text { Depth (mbsf): }\end{array}$ & $\begin{array}{c}832 \mathrm{~A} \\
1 \mathrm{H}-4 \\
54-56 \\
4.90\end{array}$ & $\begin{array}{c}832 \mathrm{~A} \\
1 \mathrm{H}-4 \\
54-56 \\
4.90\end{array}$ & $\begin{array}{c}832 \mathrm{~A} \\
1 \mathrm{H}-4 \\
54-56 \\
4.90\end{array}$ & $\begin{array}{c}832 \mathrm{~A} \\
1 \mathrm{H}-4 \\
54-56 \\
4.90\end{array}$ & $\begin{array}{c}832 \mathrm{~A} \\
1 \mathrm{H}-4 \\
54-56 \\
4.90\end{array}$ & $\begin{array}{c}832 \mathrm{~A} \\
1 \mathrm{H}-4 \\
54-56 \\
4.90\end{array}$ & $\begin{array}{c}832 \mathrm{~A} \\
1 \mathrm{H}-4 \\
54-56 \\
4.90\end{array}$ & $\begin{array}{c}832 \mathrm{~A} \\
1 \mathrm{H}-4 \\
54-56 \\
4.90\end{array}$ & $\begin{array}{c}832 \mathrm{~A} \\
1 \mathrm{H}-4 \\
54-56 \\
4.90\end{array}$ & $\begin{array}{c}832 \mathrm{~A} \\
1 \mathrm{H}-4 \\
54-56 \\
4.90\end{array}$ & $\begin{array}{c}832 \mathrm{~A} \\
1 \mathrm{H}-4 \\
54-56 \\
4.90\end{array}$ & $\begin{array}{c}832 \mathrm{~A} \\
1 \mathrm{H}-4 \\
54-56 \\
4.90\end{array}$ & $\begin{array}{c}832 \mathrm{~A} \\
1 \mathrm{H}-4 \\
54-56 \\
4.90\end{array}$ \\
\hline $\begin{array}{l}\mathrm{SiO}_{2} \\
\mathrm{TiO}_{2} \\
\mathrm{Al}_{2} \mathrm{O}_{3} \\
\mathrm{FeO} \\
\mathrm{MnO} \\
\mathrm{MgO} \\
\mathrm{CaO} \\
\mathrm{Na}_{2} \mathrm{O} \\
\mathrm{K}_{2} \mathrm{O}\end{array}$ & $\begin{array}{r}59.84 \\
0.96 \\
16.14 \\
7.05 \\
0.28 \\
1.96 \\
4.51 \\
4.56 \\
4.70\end{array}$ & $\begin{array}{r}52.88 \\
0.93 \\
16.16 \\
10.68 \\
0.26 \\
4.12 \\
8.75 \\
3.56 \\
2.66\end{array}$ & $\begin{array}{r}59.37 \\
0.92 \\
16.40 \\
7.72 \\
0.15 \\
2.05 \\
4.94 \\
4.22 \\
4.23\end{array}$ & $\begin{array}{r}62.17 \\
0.90 \\
16.23 \\
6.92 \\
0.36 \\
1.55 \\
3.72 \\
3.42 \\
4.72\end{array}$ & $\begin{array}{r}51.46 \\
1.03 \\
14.77 \\
11.72 \\
0.14 \\
5.40 \\
10.44 \\
3.28 \\
1.75\end{array}$ & $\begin{array}{r}59.40 \\
0.89 \\
16.25 \\
7.72 \\
0.37 \\
2.04 \\
4.93 \\
3.79 \\
4.61\end{array}$ & $\begin{array}{r}58.47 \\
0.86 \\
16.60 \\
8.56 \\
0.17 \\
2.20 \\
5.62 \\
3.70 \\
3.81\end{array}$ & $\begin{array}{r}52.74 \\
0.90 \\
14.13 \\
11.64 \\
0.24 \\
6.10 \\
10.80 \\
2.13 \\
1.32\end{array}$ & $\begin{array}{r}59.75 \\
0.93 \\
16.18 \\
8.01 \\
0.22 \\
1.98 \\
4.83 \\
3.58 \\
4.52\end{array}$ & $\begin{array}{r}58.50 \\
0.92 \\
16.31 \\
8.53 \\
0.27 \\
2.29 \\
5.59 \\
3.76 \\
3.85\end{array}$ & $\begin{array}{r}59.14 \\
0.98 \\
16.59 \\
7.75 \\
0.12 \\
2.17 \\
5.19 \\
4.07 \\
3.98\end{array}$ & $\begin{array}{r}60.07 \\
0.88 \\
15.31 \\
8.04 \\
0.37 \\
1.97 \\
4.76 \\
3.63 \\
4.98\end{array}$ & $\begin{array}{r}60.59 \\
0.96 \\
16.23 \\
7.16 \\
0.28 \\
1.85 \\
4.53 \\
3.93 \\
4.46\end{array}$ \\
\hline Original Total & 97.79 & 97.66 & 98.11 & 98.43 & 97.01 & 98.88 & 98.92 & 99.40 & 98.13 & 97.93 & 98.71 & 99.19 & 99.30 \\
\hline
\end{tabular}

Table 5 (continued).

\begin{tabular}{|c|c|c|c|c|c|c|c|c|c|c|c|c|c|}
\hline $\begin{array}{l}\text { Hole: } \\
\text { Core, section: } \\
\text { Interval (cm): } \\
\text { Depth (mbsf): }\end{array}$ & $\begin{array}{c}832 \mathrm{~A} \\
1 \mathrm{H}-4 \\
54-56 \\
4.90\end{array}$ & $\begin{array}{c}832 \mathrm{~A} \\
1 \mathrm{H}-4 \\
54-56 \\
4.90\end{array}$ & $\begin{array}{c}832 \mathrm{~A} \\
1 \mathrm{H}-4 \\
54-56 \\
4.90\end{array}$ & $\begin{array}{c}832 \mathrm{~A} \\
1 \mathrm{H}-4 \\
54-56 \\
4.90\end{array}$ & $\begin{array}{c}832 \mathrm{~A} \\
1 \mathrm{H}-4 \\
54-56 \\
4.90\end{array}$ & $\begin{array}{c}832 \mathrm{~A} \\
3 \mathrm{H}-1 \\
36-38 \\
15.77\end{array}$ & $\begin{array}{l}832 \mathrm{~A} \\
3 \mathrm{H}-1 \\
36-38 \\
15.77\end{array}$ & $\begin{array}{l}832 \mathrm{~A} \\
3 \mathrm{H}-1 \\
36-38 \\
15.77\end{array}$ & $\begin{array}{l}832 \mathrm{~A} \\
3 \mathrm{H}-1 \\
36-38 \\
15.77\end{array}$ & $\begin{array}{l}832 \mathrm{~A} \\
3 \mathrm{H}-1 \\
36-38 \\
15.77\end{array}$ & $\begin{array}{l}832 \mathrm{~A} \\
3 \mathrm{H}-1 \\
36-38 \\
15.77\end{array}$ & $\begin{array}{l}832 \mathrm{~A} \\
3 \mathrm{H}-1 \\
36-38 \\
15.77\end{array}$ & $\begin{array}{c}832 \mathrm{~A} \\
3 \mathrm{H}-1 \\
36-38 \\
15.77\end{array}$ \\
\hline $\begin{array}{l}\mathrm{SiO}_{2} \\
\mathrm{TiO}_{2} \\
\mathrm{Al}_{2} \mathrm{O}_{3} \\
\mathrm{FeO} \\
\mathrm{MnO} \\
\mathrm{MgO} \\
\mathrm{CaO} \\
\mathrm{Na}_{2} \mathrm{O} \\
\mathrm{K}_{2} \mathrm{O}\end{array}$ & $\begin{array}{r}60.12 \\
0.92 \\
15.40 \\
8.35 \\
0.17 \\
2.06 \\
4.30 \\
3.56 \\
5.11\end{array}$ & $\begin{array}{r}50.23 \\
0.77 \\
15.29 \\
10.93 \\
0.87 \\
6.15 \\
12.08 \\
2.46 \\
1.23\end{array}$ & $\begin{array}{r}55.81 \\
1.00 \\
16.38 \\
9.12 \\
0.15 \\
3.30 \\
7.08 \\
3.73 \\
3.43\end{array}$ & $\begin{array}{r}57.33 \\
0.98 \\
15.76 \\
9.35 \\
0.16 \\
2.46 \\
5.71 \\
4.03 \\
4.21\end{array}$ & $\begin{array}{r}60.09 \\
0.82 \\
16.33 \\
7.43 \\
0.29 \\
1.97 \\
4.86 \\
3.75 \\
4.45\end{array}$ & $\begin{array}{r}56.27 \\
1.05 \\
15.40 \\
10.51 \\
0.35 \\
2.44 \\
6.05 \\
4.08 \\
3.85\end{array}$ & $\begin{array}{r}60.11 \\
0.97 \\
16.93 \\
7.09 \\
0.33 \\
1.96 \\
4.58 \\
4.09 \\
3.95\end{array}$ & $\begin{array}{r}60.03 \\
0.94 \\
17.17 \\
7.01 \\
0.32 \\
1.90 \\
4.91 \\
3.93 \\
3.79\end{array}$ & $\begin{array}{r}54.89 \\
0.94 \\
16.25 \\
9.95 \\
0.12 \\
3.39 \\
7.43 \\
4.10 \\
2.92\end{array}$ & $\begin{array}{r}55.07 \\
1.20 \\
15.84 \\
9.94 \\
0.28 \\
3.51 \\
6.90 \\
3.85 \\
3.42\end{array}$ & $\begin{array}{r}56.16 \\
1.16 \\
15.22 \\
10.36 \\
0.31 \\
2.71 \\
6.06 \\
4.01 \\
4.01\end{array}$ & $\begin{array}{r}56.17 \\
1.23 \\
15.54 \\
10.18 \\
0.21 \\
2.88 \\
6.16 \\
3.97 \\
3.67\end{array}$ & $\begin{array}{r}54.22 \\
0.97 \\
16.54 \\
9.85 \\
0.36 \\
3.47 \\
7.68 \\
4.07 \\
2.83\end{array}$ \\
\hline Original Total & 99.02 & 98.71 & 98.60 & 99.43 & 98.37 & 100.83 & 100.10 & 99.77 & 99.91 & 97.74 & 99.80 & 99.31 & 99.17 \\
\hline
\end{tabular}

Table 5 (continued).

\begin{tabular}{|c|c|c|c|c|c|c|c|c|c|c|c|c|c|}
\hline $\begin{array}{l}\text { Hole: } \\
\text { Core, section: } \\
\text { Interval }(\mathrm{cm}): \\
\text { Depth (mbsf): }\end{array}$ & $\begin{array}{l}832 \mathrm{~A} \\
3 \mathrm{H}-1 \\
36-38 \\
15.77\end{array}$ & $\begin{array}{l}832 \mathrm{~A} \\
3 \mathrm{H}-1 \\
36-38 \\
15.77\end{array}$ & $\begin{array}{c}832 \mathrm{~A} \\
3 \mathrm{H}-1 \\
36-38 \\
15.77\end{array}$ & $\begin{array}{l}832 \mathrm{~A} \\
3 \mathrm{H}-1 \\
36-38 \\
15.77\end{array}$ & $\begin{array}{c}832 \mathrm{~A} \\
3 \mathrm{H}-1 \\
36-38 \\
15.77\end{array}$ & $\begin{array}{c}832 \mathrm{~A} \\
10 \mathrm{H}-1 \\
120-122 \\
73.71\end{array}$ & $\begin{array}{c}832 \mathrm{~A} \\
10 \mathrm{H}-1 \\
120-122 \\
73.71\end{array}$ & $\begin{array}{c}832 \mathrm{~A} \\
10 \mathrm{H}-1 \\
120-122 \\
73.71\end{array}$ & $\begin{array}{l}832 \mathrm{~A} \\
10 \mathrm{H}-1 \\
120-122 \\
73.71\end{array}$ & $\begin{array}{c}832 \mathrm{~A} \\
10 \mathrm{H}-1 \\
120-122 \\
73.71\end{array}$ & $\begin{array}{c}832 \mathrm{~A} \\
10 \mathrm{H}-1 \\
120-122 \\
73.71\end{array}$ & $\begin{array}{c}832 \mathrm{~A} \\
10 \mathrm{H}-1 \\
120-122 \\
73.71\end{array}$ & $\begin{array}{c}832 \mathrm{~A} \\
10 \mathrm{H}-1 \\
120-122 \\
73.71\end{array}$ \\
\hline $\begin{array}{l}\mathrm{SiO}_{2} \\
\mathrm{TiO}_{2} \\
\mathrm{Al}_{2} \mathrm{O}_{3} \\
\mathrm{FeO} \\
\mathrm{MnO} \\
\mathrm{MgO} \\
\mathrm{CaO} \\
\mathrm{Na}_{2} \mathrm{O} \\
\mathrm{K}_{2} \mathrm{O}\end{array}$ & $\begin{array}{r}55.02 \\
1.10 \\
15.26 \\
10.42 \\
0.32 \\
3.23 \\
7.28 \\
3.94 \\
3.43\end{array}$ & $\begin{array}{r}60.78 \\
0.68 \\
17.51 \\
5.36 \\
0.17 \\
1.75 \\
4.17 \\
4.97 \\
4.60\end{array}$ & $\begin{array}{r}56.98 \\
1.01 \\
15.70 \\
9.33 \\
0.16 \\
2.77 \\
6.22 \\
4.43 \\
3.40\end{array}$ & $\begin{array}{r}57.43 \\
1.13 \\
15.18 \\
9.36 \\
0.16 \\
2.65 \\
5.53 \\
4.55 \\
3.99\end{array}$ & $\begin{array}{r}54.46 \\
1.10 \\
15.66 \\
10.12 \\
0.44 \\
3.41 \\
7.20 \\
3.66 \\
3.94\end{array}$ & $\begin{array}{r}63.70 \\
0.91 \\
14.33 \\
8.33 \\
0.25 \\
1.84 \\
4.90 \\
3.10 \\
2.64\end{array}$ & $\begin{array}{r}49.98 \\
1.01 \\
16.11 \\
11.17 \\
0.34 \\
4.84 \\
10.82 \\
3.57 \\
2.17\end{array}$ & $\begin{array}{r}49.90 \\
0.80 \\
15.85 \\
11.46 \\
0.20 \\
5.21 \\
11.46 \\
3.17 \\
1.96\end{array}$ & $\begin{array}{r}55.51 \\
0.96 \\
14.50 \\
11.84 \\
0.23 \\
3.95 \\
8.65 \\
3.09 \\
1.28\end{array}$ & $\begin{array}{r}71.37 \\
0.46 \\
14.89 \\
3.06 \\
0.02 \\
0.48 \\
1.76 \\
3.57 \\
4.39\end{array}$ & $\begin{array}{r}50.30 \\
0.95 \\
15.02 \\
11.91 \\
0.28 \\
5.27 \\
10.37 \\
3.50 \\
2.39\end{array}$ & $\begin{array}{r}50.35 \\
0.97 \\
15.68 \\
11.65 \\
0.29 \\
4.84 \\
10.46 \\
3.51 \\
2.26\end{array}$ & $\begin{array}{r}69.81 \\
0.43 \\
14.92 \\
3.82 \\
0.21 \\
0.65 \\
3.15 \\
3.92 \\
3.09\end{array}$ \\
\hline Original Total & 98.82 & 100.34 & 98.36 & 98.88 & 99.65 & 95.85 & 95.32 & 96.42 & 96.98 & 95.09 & 95.43 & 96.11 & 94.42 \\
\hline
\end{tabular}

Table 5 (continued).

\begin{tabular}{|c|c|c|c|c|c|c|c|c|c|c|c|c|c|}
\hline $\begin{array}{l}\text { Hole: } \\
\text { Core, section: } \\
\text { Interval (cm): } \\
\text { Depth (mbsf): }\end{array}$ & $\begin{array}{c}832 \mathrm{~A} \\
10 \mathrm{H}-1 \\
120-122 \\
73.71\end{array}$ & $\begin{array}{c}832 \mathrm{~A} \\
10 \mathrm{H}-1 \\
120-122 \\
73.71\end{array}$ & $\begin{array}{c}832 \mathrm{~A} \\
10 \mathrm{H}-1 \\
120-122 \\
73.71\end{array}$ & $\begin{array}{c}832 \mathrm{~A} \\
10 \mathrm{H}-1 \\
120-122 \\
73.71\end{array}$ & $\begin{array}{c}832 \mathrm{~A} \\
10 \mathrm{H}-1 \\
120-122 \\
73.71\end{array}$ & $\begin{array}{c}832 \mathrm{~A} \\
10 \mathrm{H}-1 \\
120-122 \\
73.71\end{array}$ & $\begin{array}{l}832 \mathrm{~A} \\
20 \mathrm{H}-1 \\
80-82 \\
145.80\end{array}$ & $\begin{array}{l}832 \mathrm{~A} \\
20 \mathrm{H}-1 \\
80-82 \\
145.80\end{array}$ & $\begin{array}{r}832 \mathrm{~A} \\
20 \mathrm{H}-1 \\
80-82 \\
145.80\end{array}$ & $\begin{array}{l}832 \mathrm{~A} \\
20 \mathrm{H}-1 \\
80-82 \\
145.80\end{array}$ & $\begin{array}{l}832 \mathrm{~A} \\
20 \mathrm{H}-1 \\
80-82 \\
145.80\end{array}$ & $\begin{array}{l}832 \mathrm{~A} \\
20 \mathrm{H}-1 \\
80-82 \\
145.80\end{array}$ & $\begin{array}{l}832 \mathrm{~A} \\
20 \mathrm{H}-1 \\
80-82 \\
145.80\end{array}$ \\
\hline $\begin{array}{l}\mathrm{SiO}_{2} \\
\mathrm{TiO}_{2} \\
\mathrm{Al}_{2} \mathrm{O}_{3} \\
\mathrm{FeO} \\
\mathrm{MnO} \\
\mathrm{MgO} \\
\mathrm{CaO} \\
\mathrm{Na}_{2} \mathrm{O} \\
\mathrm{K}_{2} \mathrm{O}\end{array}$ & $\begin{array}{r}49.78 \\
0.88 \\
15.35 \\
12.70 \\
0.14 \\
4.93 \\
10.58 \\
3.39 \\
2.26\end{array}$ & $\begin{array}{r}65.88 \\
0.78 \\
14.63 \\
6.88 \\
0.30 \\
1.46 \\
4.31 \\
2.91 \\
2.85\end{array}$ & $\begin{array}{r}50.30 \\
0.90 \\
16.09 \\
11.26 \\
0.23 \\
4.76 \\
10.77 \\
3.42 \\
2.27\end{array}$ & $\begin{array}{r}67.18 \\
0.63 \\
15.00 \\
5.64 \\
0.21 \\
1.24 \\
3.70 \\
3.49 \\
2.90\end{array}$ & $\begin{array}{r}50.54 \\
1.03 \\
15.70 \\
11.26 \\
0.34 \\
4.82 \\
9.91 \\
3.84 \\
2.57\end{array}$ & $\begin{array}{r}51.33 \\
0.95 \\
15.95 \\
11.46 \\
0.30 \\
4.13 \\
9.29 \\
3.97 \\
2.61\end{array}$ & $\begin{array}{r}53.45 \\
0.95 \\
16.15 \\
10.15 \\
0.09 \\
4.04 \\
8.85 \\
3.51 \\
2.81\end{array}$ & $\begin{array}{r}53.24 \\
1.02 \\
16.57 \\
10.08 \\
0.25 \\
3.92 \\
8.67 \\
3.55 \\
2.69\end{array}$ & $\begin{array}{r}50.60 \\
0.97 \\
15.74 \\
10.49 \\
0.14 \\
5.47 \\
10.92 \\
3.50 \\
2.18\end{array}$ & $\begin{array}{r}51.56 \\
1.08 \\
16.33 \\
10.70 \\
0.24 \\
4.57 \\
9.88 \\
3.31 \\
2.33\end{array}$ & $\begin{array}{r}51.05 \\
1.20 \\
15.16 \\
11.93 \\
0.22 \\
4.67 \\
9.55 \\
3.75 \\
2.46\end{array}$ & $\begin{array}{r}50.60 \\
0.98 \\
15.26 \\
11.51 \\
0.34 \\
5.26 \\
10.02 \\
3.61 \\
2.41\end{array}$ & $\begin{array}{r}50.10 \\
1.10 \\
15.03 \\
11.37 \\
0.18 \\
5.92 \\
11.32 \\
3.07 \\
1.91\end{array}$ \\
\hline Original Total & 96.21 & 95.17 & 95.55 & 97.78 & 95.39 & 95.43 & 96.58 & 96.50 & 97.47 & 97.18 & 98.32 & 96.93 & 96.93 \\
\hline
\end{tabular}


Table 5 (continued).

\begin{tabular}{|c|c|c|c|c|c|c|c|c|c|c|c|c|c|}
\hline $\begin{array}{l}\text { Hole: } \\
\text { Core, section: } \\
\text { Interval }(\mathrm{cm}) \text { : } \\
\text { Depth (mbsf): }\end{array}$ & $\begin{array}{l}832 \mathrm{~A} \\
20 \mathrm{H}-1 \\
80-82 \\
145.80\end{array}$ & $\begin{array}{l}832 \mathrm{~A} \\
20 \mathrm{H}-1 \\
80-82 \\
145.80\end{array}$ & $\begin{array}{l}832 \mathrm{~A} \\
20 \mathrm{H}-1 \\
80-82 \\
145.80\end{array}$ & $\begin{array}{l}832 \mathrm{~A} \\
20 \mathrm{H}-1 \\
80-82 \\
145.80\end{array}$ & $\begin{array}{l}832 \mathrm{~A} \\
20 \mathrm{H}-1 \\
80-82 \\
145.80\end{array}$ & $\begin{array}{l}832 \mathrm{~A} \\
20 \mathrm{H}-1 \\
80-82 \\
145.80\end{array}$ & $\begin{array}{l}832 \mathrm{~A} \\
20 \mathrm{H}-1 \\
80-82 \\
145.80\end{array}$ & $\begin{array}{c}832 \mathrm{~A} \\
20 \mathrm{H}-1 \\
80-82 \\
145.80\end{array}$ & $\begin{array}{l}832 \mathrm{~A} \\
20 \mathrm{H}-1 \\
80-82 \\
145.80\end{array}$ & $\begin{array}{c}833 \mathrm{~A} \\
1 \mathrm{H}-6 \\
72-74 \\
8.18\end{array}$ & $\begin{array}{c}833 \mathrm{~A} \\
1 \mathrm{H}-6 \\
72-74 \\
8.18\end{array}$ & $\begin{array}{c}833 \mathrm{~A} \\
1 \mathrm{H}-6 \\
72-74 \\
8.18\end{array}$ & $\begin{array}{c}833 \mathrm{~A} \\
1 \mathrm{H}-6 \\
72-74 \\
8.18\end{array}$ \\
\hline $\begin{array}{l}\mathrm{SiO}_{2} \\
\mathrm{TiO}_{2} \\
\mathrm{Al}_{2} \mathrm{O}_{3} \\
\mathrm{FeO} \\
\mathrm{MnO} \\
\mathrm{MgO} \\
\mathrm{CaO} \\
\mathrm{Na}_{2} \mathrm{O} \\
\mathrm{K}_{2} \mathrm{O}\end{array}$ & $\begin{array}{r}50.59 \\
0.94 \\
15.44 \\
10.79 \\
0.26 \\
5.39 \\
11.53 \\
3.05 \\
2.01\end{array}$ & $\begin{array}{r}50.46 \\
0.98 \\
15.81 \\
10.12 \\
0.17 \\
5.84 \\
11.38 \\
3.30 \\
1.92\end{array}$ & $\begin{array}{r}50.27 \\
0.89 \\
15.46 \\
10.50 \\
0.27 \\
5.69 \\
11.56 \\
3.33 \\
2.04\end{array}$ & $\begin{array}{r}52.79 \\
1.17 \\
15.02 \\
11.49 \\
0.28 \\
3.76 \\
8.46 \\
3.58 \\
3.46\end{array}$ & $\begin{array}{r}50.51 \\
0.92 \\
15.59 \\
10.90 \\
0.25 \\
5.30 \\
11.17 \\
3.26 \\
2.11\end{array}$ & $\begin{array}{r}50.25 \\
0.98 \\
15.40 \\
10.63 \\
0.23 \\
6.06 \\
12.06 \\
2.89 \\
1.49\end{array}$ & $\begin{array}{r}51.42 \\
1.06 \\
16.35 \\
10.64 \\
0.22 \\
4.83 \\
9.90 \\
3.23 \\
2.35\end{array}$ & $\begin{array}{r}50.46 \\
1.27 \\
15.14 \\
11.60 \\
0.15 \\
5.55 \\
10.57 \\
3.23 \\
2.02\end{array}$ & $\begin{array}{r}50.06 \\
0.90 \\
15.51 \\
11.21 \\
0.22 \\
5.53 \\
11.58 \\
3.03 \\
1.96\end{array}$ & $\begin{array}{r}51.80 \\
1.18 \\
16.13 \\
10.54 \\
0.15 \\
4.84 \\
9.84 \\
3.29 \\
2.23\end{array}$ & $\begin{array}{r}51.45 \\
1.09 \\
14.97 \\
11.25 \\
0.21 \\
5.29 \\
9.85 \\
3.37 \\
2.53\end{array}$ & $\begin{array}{r}55.06 \\
0.81 \\
16.97 \\
8.74 \\
0.22 \\
3.36 \\
7.36 \\
3.78 \\
3.69\end{array}$ & $\begin{array}{r}54.32 \\
0.88 \\
16.18 \\
9.61 \\
0.17 \\
4.22 \\
9.43 \\
3.41 \\
1.77\end{array}$ \\
\hline Original Total & 97.45 & 98.39 & 97.36 & 97.30 & 97.91 & 98.36 & 96.46 & 95.89 & 96.56 & 96.93 & 97.28 & 98.09 & 98.10 \\
\hline
\end{tabular}

Table 5 (continued).

\begin{tabular}{|c|c|c|c|c|c|c|c|c|c|c|c|c|c|}
\hline $\begin{array}{l}\text { Hole: } \\
\text { Core, section: } \\
\text { Interval (cm): } \\
\text { Depth (mbsf): }\end{array}$ & $\begin{array}{c}833 \mathrm{~A} \\
1 \mathrm{H}-6 \\
72-74 \\
8.18\end{array}$ & $\begin{array}{c}833 \mathrm{~A} \\
1 \mathrm{H}-6 \\
72-74 \\
8.18\end{array}$ & $\begin{array}{c}833 \mathrm{~A} \\
1 \mathrm{H}-6 \\
72-74 \\
8.18\end{array}$ & $\begin{array}{c}833 \mathrm{~A} \\
1 \mathrm{H}-6 \\
72-74 \\
8.18\end{array}$ & $\begin{array}{c}833 \mathrm{~A} \\
1 \mathrm{H}-6 \\
72-74 \\
8.18\end{array}$ & $\begin{array}{c}833 \mathrm{~A} \\
1 \mathrm{H}-6 \\
72-74 \\
8.18\end{array}$ & $\begin{array}{c}833 \mathrm{~A} \\
1 \mathrm{H}-6 \\
72-74 \\
8.18\end{array}$ & $\begin{array}{c}833 \mathrm{~A} \\
1 \mathrm{H}-6 \\
72-74 \\
8.18\end{array}$ & $\begin{array}{c}833 \mathrm{~A} \\
1 \mathrm{H}-6 \\
72-74 \\
8.18\end{array}$ & $\begin{array}{c}833 \mathrm{~A} \\
1 \mathrm{H}-6 \\
72-74 \\
8.18\end{array}$ & $\begin{array}{c}833 \mathrm{~A} \\
1 \mathrm{H}-6 \\
72-74 \\
8.18\end{array}$ & $\begin{array}{c}833 \mathrm{~A} \\
1 \mathrm{H}-6 \\
72-74 \\
8.18\end{array}$ & $\begin{array}{c}833 \mathrm{~A} \\
1 \mathrm{H}-6 \\
72-74 \\
8.18\end{array}$ \\
\hline $\begin{array}{l}\mathrm{SiO}_{2} \\
\mathrm{TiO}_{2} \\
\mathrm{Al}_{2} \mathrm{O}_{3} \\
\mathrm{FeO} \\
\mathrm{MnO} \\
\mathrm{MgO} \\
\mathrm{CaO} \\
\mathrm{Na}_{2} \mathrm{O} \\
\mathrm{K}_{2} \mathrm{O}\end{array}$ & $\begin{array}{r}52.62 \\
1.00 \\
15.85 \\
10.17 \\
0.39 \\
4.76 \\
9.72 \\
3.17 \\
2.32\end{array}$ & $\begin{array}{r}55.08 \\
1.04 \\
15.95 \\
9.84 \\
0.11 \\
3.48 \\
7.17 \\
3.76 \\
3.57\end{array}$ & $\begin{array}{r}55.50 \\
0.90 \\
16.22 \\
9.25 \\
0.34 \\
3.35 \\
6.79 \\
3.57 \\
4.06\end{array}$ & $\begin{array}{r}52.83 \\
1.09 \\
16.26 \\
10.04 \\
0.17 \\
4.34 \\
9.26 \\
3.37 \\
2.64\end{array}$ & $\begin{array}{r}54.93 \\
0.97 \\
17.04 \\
8.25 \\
0.22 \\
3.34 \\
7.69 \\
3.90 \\
3.66\end{array}$ & $\begin{array}{r}53.19 \\
1.02 \\
15.75 \\
10.13 \\
0.19 \\
4.39 \\
9.69 \\
3.25 \\
2.40\end{array}$ & $\begin{array}{r}56.87 \\
0.90 \\
17.88 \\
7.60 \\
0.21 \\
2.44 \\
6.33 \\
3.62 \\
4.15\end{array}$ & $\begin{array}{r}56.63 \\
1.05 \\
16.26 \\
8.69 \\
0.25 \\
2.79 \\
5.95 \\
3.91 \\
4.47\end{array}$ & $\begin{array}{r}52.94 \\
1.07 \\
16.16 \\
9.82 \\
0.32 \\
4.34 \\
9.35 \\
3.42 \\
2.57\end{array}$ & $\begin{array}{r}55.37 \\
0.91 \\
16.83 \\
8.31 \\
0.26 \\
3.37 \\
7.68 \\
3.67 \\
3.60\end{array}$ & $\begin{array}{r}52.84 \\
0.95 \\
16.39 \\
9.75 \\
0.26 \\
4.53 \\
9.52 \\
3.33 \\
2.43\end{array}$ & $\begin{array}{r}56.92 \\
0.98 \\
16.44 \\
8.19 \\
0.25 \\
3.10 \\
6.51 \\
3.66 \\
3.96\end{array}$ & $\begin{array}{r}52.39 \\
1.08 \\
15.77 \\
10.25 \\
0.32 \\
4.49 \\
9.62 \\
3.64 \\
2.44\end{array}$ \\
\hline Original Total & 98.14 & 97.96 & 97.47 & 97.74 & 98.11 & 96.23 & 96.95 & 98.17 & 97.01 & 97.51 & 97.12 & 96.28 & 96.42 \\
\hline
\end{tabular}

Table 5 (continued).

\begin{tabular}{lcrrr}
\hline Hole: & $833 \mathrm{~A}$ & $833 \mathrm{~A}$ & $833 \mathrm{~A}$ & \multicolumn{1}{c}{$833 \mathrm{~A}$} \\
Core, section: & $1 \mathrm{H}-6$ & $1 \mathrm{H}-6$ & $25 \mathrm{R}-2$ & $25 \mathrm{R}-2$ \\
Interval (cm): & $72-74$ & $72-74$ & $29-31$ & $29-31$ \\
Depth (mbsf): & 8.18 & 8.18 & 290.70 & 290.70 \\
\hline & & & & \\
\hline $\mathrm{SiO}_{2}$ & 53.90 & 55.68 & 54.81 & 56.42 \\
$\mathrm{TiO}_{2}$ & 1.27 & 0.80 & 0.70 & 0.61 \\
$\mathrm{Al}_{2} \mathrm{O}_{3}$ & 14.65 & 16.85 & 8.83 & 8.15 \\
$\mathrm{FeO}$ & 11.49 & 8.65 & 14.26 & 13.97 \\
$\mathrm{MnO}$ & 0.41 & 0.21 & 0.08 & 0.22 \\
$\mathrm{MgO}$ & 3.71 & 3.29 & 15.13 & 16.36 \\
$\mathrm{CaO}$ & 7.82 & 7.22 & 4.68 & 3.48 \\
$\mathrm{Na} \mathrm{O}_{2} \mathrm{O}$ & 3.56 & 3.54 & 1.20 & 0.62 \\
$\mathrm{~K} 2 \mathrm{O}$ & 3.20 & 3.76 & 0.30 & 0.17 \\
Original Total & 97.30 & 97.43 & 86.73 & 82.19 \\
\hline
\end{tabular}

Note: For comparative purposes analyses have been recalculated to $100 \%$. "Original total" indicates the raw total returned by electron microprobe analysis. Total Fe is reported as FeO. Columns showing the same sample number refer to analyses of different glass shards in the same ash specimen.

ple $134-828 \mathrm{~A}-4 \mathrm{H}-2,55-57 \mathrm{~cm}$.). It is also weakly represented in the North Aoba Basin (Sample 134-832A-1H-4, 54-56 cm.) This is again strongly suggestive of an event in the Lopevi-Epi-Tongoa group where explosive eruptions belonging to the lower-K trend are known to have occurred during the Quaternary (Crawford et al.,1988).

3. The main group of glasses on the $\mathrm{K}_{2} \mathrm{O}$ versus $\mathrm{SiO}_{2}$ diagram (Fig. 4) conform to the high-K trend and are strongly represented in all the North Aoba Basin samples. Glass of this type appears only at one of the forearc sites, on the NDR (Sample 134-828A-2H-6, 7-9 $\mathrm{cm}$ ). The Central Basin volcanoes of Ambrym, Aoba, and Santa Maria are the most likely source of these ashes. The $\mathrm{K}_{2} \mathrm{O}-\mathrm{SiO}_{2}$ trend on Figure 4 coincides with the trends for the latter two islands illustrated by Macfarlane et al. (1988, their Fig. 19). The more differentiated glasses in Sample 134-832A-4H, 54-56 cm, have almost certainly originated from Santa Maria as Ambrym and Aoba are restricted to more basic compositions (Macfarlane et al., 1988). It is apparently rare, but not unknown, for small quantities of ash from these eruptions to reach the DEZ some $150 \mathrm{~km}$ west of the Central Chain.

4. Sample 134-832A-10H-1, 120-122 cm, is notable in that it contains glass of two quite distinct compositions, one a high-K basalt and the other a low-K dacite. It is likely that this represents a combined input from two separate events, the high- $\mathrm{K}$ basalt coming from perhaps Santa Maria or Aoba and the low-K dacite from the Lopevi-EpiTongoa group.

5. Mineral compositions have not proved helpful in distinguishing the source of the ashes. Olivine compositions from the Leg 134 ashes fall within the ranges reported for the Central Chain volcanoes of the Epi group (Crawford et al., 1988; Bardsell and Berry, 1990) and are also similar to those reported for Merelava (Bardsell, 1988). However, the ashes from the drill sites apparently lack the Ca-poor 
clinopyroxenes reported from these other locations. Plagioclase from the Leg 134 samples shows an almost identical compositional range to that in the Epi group and Merelava rocks.

\section{CONCLUSIONS}

Crystalline constituents in the Leg 134 ashes are plagioclase, clinopyroxene, olivine, magnetite, titanomagnetite, and rarely orthopyroxene, apatite, and quartz. Most of the ashes also contain glass shards, which often span a wide compositional range in a single thin layer. The overall compositional spread is from basalt to rhyolite (on a water-free basis). The composite nature of the ash layers is probably best accounted for by the faster accumulation of ashes from separate eruptions or eruptive phases relative to the slow background sedimentation rate. The distinct high- $\mathrm{K}$ and low- $\mathrm{K}$ trends previously recognized in the Vanuatu volcanic rocks (Macfarlane et al., 1988) are also distinguished in the Leg 134 glasses. The high-K trend is abundantly represented in ashes from the North Aoba Basin sites and the source is most likely to be the Central Basin volcanoes of Santa Maria, Aoba, and Ambrym. Highly potassic glass of this type is not observed at the forearc sites apart from in one sample from the NDR. A low-K trend is dominant amongst glasses from the forearc sites and in one of the North Aoba Basin ashes. Medium-K dacite ash features at Site 830. The low- and medium-K glasses have probably been derived from eruptions in the Lopevi-Epi-Tongoa group, associated with caldera subsidence. During the time span of the ash sequence investigated there is no evidence of any systematic compositional change: high- and low-K magmas appear to have been available throughout. The distribution of the pyroclastics was controlled principally by the upper westerly winds, with the low-level southeast trades exerting a minor influence.

\section{ACKNOWLEDGMENTS}

We are grateful for the constructive criticism and helpful comments received from Prof. R.J. Arculus, Prof. H. Colley, Dr. A.J. Crawford, and Dr. H.G. Greene. We thank Dr. J. Rosenbaum for help with pyroxene geothermometry.

\section{REFERENCES}

Arculus, R.J., and Bloomfield, A.L., 1992. Major-element geochemistry of ashes from Sites 782, 784, and 786 in the Bonin forearc. In Fryer, P., Pearce,
J.A., Stokking, L.B., et al., Proc. ODP, Sci. Results, 125: College Station, TX (Ocean Drilling Program), 277-292.

Bardsell, M., 1988. Petrology and petrogenesis of clinopyroxene-rich tholeiitic lavas, Merelava Volcano, Vanuatu. J. Petrol., 29:927-964.

Bardsell, M., and Berry, R.F., 1990. Origin and evolution of primitive island arc ankaramites from western Epi, Vanuatu. J. Petrol., 31:747-777.

Crawford, A.J., Greene, H.G., and Exon, N.J., 1988. Geology, petrology and geochemistry of submarine volcanoes around Epi Island, New Hebrides Island Arc. In Greene, H.G., and Wong, F.L. (Eds.), Geology and Offshore Resources of Pacific Island Arcs-Vanuatu Region. Circum-Pac. Counc. Energy Miner. Resour., Earth Sci. Ser., 8:301-327.

Eade, J.V., and Gregory, M.R., 1992. Sediments of the North Fiji Basin. In Kroenke, L.W., and Eade, J.V. (Eds.), Basin Formation, Ridge Crest Processes and Metallogenesis in the North Fiji Basin. Circum-Pac. Counc. Energy Miner. Resour., Earth Sci. Ser., 9:97-126.

Gorton, M.P., 1977. The geochemistry and origin of Quaternary volcanism in the New Hebrides. Geochim. Cosmochim. Acta, 41:1257-1270.

Jezek, P.A., 1976. Compositional variations within and among volcanic ash layers in the Fiji Plateau area. J. Geol., 84:595-616.

Lindsley, D.H., and Anderson, D.J., 1983. A two-pyroxene thermometer. $J$. Geophys. Res., 88:A887-A906.

Macfarlane, A., Carney, J.N., Crawford, A.J., and Greene, H.G., 1988. Vanuatu-a review of the onshore geology. In Greene, H.G., and Wong, F.L. (Eds.), Geology and Offshore Resources of Pacific Island Arcs-Vanuatu Region. Circum-Pac. Counc. Energy and Miner. Resour., Earth Sci. Ser., $8: 45-91$.

Mallick, D.I.J., 1973. Some petrological and structural variations in the New Hebrides. In Coleman, P.J. (Ed.), The Western Pacific: Island Arcs, Marginal Seas, Geochemistry: New York (Crane, Russak and Co.), 193-211.

Miyashiro, A., 1974. Volcanic rock series in island ares and active continental margins. Am. J. Sci., 274:321-355.

Robson, G.R., and Tomblin, J.F., 1966. Catalogue of the Active Volcanoes of the World Including Solfatara Fields. Pt. 20: West Indies. Int. Assoc. Volcanol.

Warden, A.J., 1967. The 1963-65 eruption of Lopevi Volcano (New Hebrides). Bull. Volcanol., 30:277-318.

\footnotetext{
Abbreviations for names of organizations and publications in ODP reference lists follow the style given in Chemical Abstracts Service Source Index (published by American Chemical Society).
}

Date of initial receipt: 27 March 1992

Date of acceptance: 7 June 1993

Ms 134SR-020 